\title{
Pathologic gene network rewiring implicates PPP1R3A as a central regulator in pressure overload heart failure
}

Pablo Cordero ${ }^{1,2,20}$, Victoria N. Parikh1,20, Elizabeth T. Chin (10 1,2, Ayca Erbilgin', Michael J. Gloudemans (1) 2,3, Ching Shang ${ }^{1}$, Yong Huang ${ }^{1}$, Alex C. Chang (1) ${ }^{1}$, Kevin S. Smith³, Frederick Dewey ${ }^{3}$, Kathia Zaleta ${ }^{1}$ Michael Morley ${ }^{4}$, Jeff Brandimarto ${ }^{5}$, Nicole Glazer ${ }^{6}$, Daryl Waggott ${ }^{1}$, Aleksandra Pavlovic ${ }^{1}$, Mingming Zhao ${ }^{7}$, Christine S. Moravec ${ }^{8}$, W.H.Wilson Tang ${ }^{8,9}$, Jamie Skreen ${ }^{10}$, Christine Malloy ${ }^{11}$, Sridhar Hannenhalli ${ }^{11}$, Hongzhe Lij ${ }^{12}$, Scott Ritter ${ }^{4}$, Mingyao Li ${ }^{12}$, Daniel Bernstein (1) ${ }^{7}$, Andrew Connolly ${ }^{13}$, Hakon Hakonarson ${ }^{14}$, Aldons J. Lusis (15) ${ }^{15}$, Kenneth B. Margulies 4,5,16, Anna A. Depaoli-Roach ${ }^{17}$, Stephen B. Montgomery (1) 3,18, Matthew T. Wheeler ${ }^{1,19}$, Thomas Cappola ${ }^{4,5,16}$ \& Euan A. Ashley (1) 1,18,19

Heart failure is a leading cause of mortality, yet our understanding of the genetic interactions underlying this disease remains incomplete. Here, we harvest 1352 healthy and failing human hearts directly from transplant center operating rooms, and obtain genome-wide genotyping and gene expression measurements for a subset of 313. We build failing and non-failing cardiac regulatory gene networks, revealing important regulators and cardiac expression quantitative trait loci (eQTLs). PPP1R3A emerges as a regulator whose network connectivity changes significantly between health and disease. RNA sequencing after PPP1R3A knockdown validates network-based predictions, and highlights metabolic pathway regulation associated with increased cardiomyocyte size and perturbed respiratory metabolism. Mice lacking PPP1R3A are protected against pressure-overload heart failure. We present a global gene interaction map of the human heart failure transition, identify previously unreported cardiac eQTLs, and demonstrate the discovery potential of disease-specific networks through the description of PPP1R3A as a central regulator in heart failure.

\footnotetext{
${ }^{1}$ Division of Cardiovascular Medicine, Stanford University, Stanford, CA 94305, USA. ${ }^{2}$ Biomedical Informatics Program, Stanford University, Stanford, CA 94305, USA. ${ }^{3}$ Department of Pathology, Stanford University, Stanford, CA 94305, USA. ${ }^{4}$ Division of Cardiology, University of Pennsylvania, Philadelphia, PA 19104, USA. ${ }^{5}$ Department of Medicine, University of Pennsylvania, Philadelphia, PA 19104, USA. ${ }^{6}$ Department of Medicine, Boston University School of Medicine, Boston, MA 02118, USA. ${ }^{7}$ Department of Pediatrics, Stanford University, Stanford, CA 94305, USA. ${ }^{8}$ Department of Cardiovascular and Metabolic Sciences, Lerner Research Institute, Cleveland, OH 44195, USA. ${ }^{9}$ Department of Cardiovascular Medicine, Heart and Vascular Institute, Cleveland, OH 44195, USA. ${ }^{10}$ Providence Medical Group-Milwaukie, Portland, OR 97222, USA. ${ }^{11}$ Center for Bioinformatics and Computational Biology, University of Maryland, College Park, MD 20740, USA. ${ }^{12}$ Biostatistics and Epidemiology, University of Pennsylvania, Philadelphia, PA 19104, USA. ${ }^{13}$ Department of Pathology, University of California San Francisco, San Francisco, CA 94143, USA. ${ }^{4}$ Department of Pediatrics, The Children's Hospital of Philadelphia, Philadelphia, PA 19104, USA. ${ }^{15}$ Departments of Medicine, University of California Los Angeles, Los Angeles, CA 90095, USA. ${ }^{16}$ Perelman School of Medicine, University of Pennsylvania, Philadelphia, PA 19104, USA. ${ }^{17}$ School of Medicine, Indiana University, Indianapolis, IN 46202, USA. ${ }^{18}$ Department of Genetics, Stanford University, Stanford, CA 94305, USA. ${ }^{19}$ Center for Undiagnosed Diseases, Stanford University, Stanford, CA 94305, USA. ${ }^{20}$ These authors contributed equally: Pablo Cordero, Victoria N. Parikh. Correspondence and requests for materials should be addressed to E.A.A. (email: euan@stanford.edu)
} 
eart failure (HF) is a life-threatening syndrome characterized by an inability of the heart to meet the metabolic demands of the body. HF costs the US more than $\$ 34$ billion a year to treat 6 million patients ${ }^{1,2}$. Despite this, the underlying molecular mechanisms remain poorly understood and the few approved therapeutics target maladaptive compensatory pathology rather than proximate molecular mechanisms ${ }^{3,4}$.

With rapidly increasing access to high-throughput sequencing technology, molecular characterization of human heart tissue has become possible, and in recent years a number of efforts to define the regulatory transcriptional architecture of HF in humans and small animals have been undertaken ${ }^{5-12}$. These efforts have revealed changes in gene expression of key sarcomeric, calcium cycling, developmental and metabolic genes. Because of the significant logistical challenge of harvesting healthy hearts, few studies have included a nonfailing control group, making conclusions regarding the transition to $\mathrm{HF}$ tentative. Further, as gene expression programs are rapidly altered in an environment of high oxidative and nitrosative stress ${ }^{13}$, the high metabolic rate of the heart limits the utility of post-mortem tissue for gene expression analysis (e.g., from public resources such as GTEx ${ }^{14-16}$ ).

Thus, the expansion of this resource with high-fidelity tissue collection and molecular characterization is required, and a crucial next step in synthesizing this information is the identification, and in vitro and in vivo validation, of novel molecular actors in this disease. In this study, we identify previously undetected cardiac expression quantitative trait loci (eQTLs) from genomewide genotyping and gene expression measurements from rapidly preserved failing and nonfailing human heart tissue. Conditionspecific cardiac regulatory gene networks identify disease-driven changes in local and global topology and pathway organization, illuminating $P P P 1 R 3 A$ as a novel predicted HF regulator. Lastly, our in vitro and in vivo approaches further demonstrate its role in HF pathology.

\section{Results}

Immediate tissue processing yields quality transcriptomic data. The MAGnet consortium was founded to establish best practices for the harvesting of human cardiac tissue (see Methods) and to explore the genetic landscape of cardiac gene expression $7,13,17$. Using this consensus protocol, we obtained 1352 human cardiac samples and chose a subset of 313 hearts, including 177 failing hearts collected immediately post transplantation and 136 healthy donor controls that were suitable for transplantation but did not reach a recipient due to logistical reasons (clinical characteristics listed in Supplementary Table 1). We genotyped and measured left-ventricular genome-wide gene expression of these samples, controlling for known covariates, specifically age, gender and collection site. Principal component analysis showed that additional covariates do not explain a significant proportion of variance in gene expression (Fig. 1a and Supplementary Fig. 1).

We assessed the quality of these measurements in several ways. First, we found that disease status was the dominant source of variation suggesting no major confounding sources of variation (Supplementary Fig. 1). Second, we confirmed enhanced expression of NPPA and NPPB, depletion of SERCA2A, and a shift from MYH7 towards MYH6 expression-established signatures of HF (Supplementary Fig. 1A and B). In total, 793 genes were significantly upregulated in failing hearts compared to nonfailing and 848 were downregulated (fold change greater or lesser than 2 or 0.5 respectively, with $\mathrm{FDR}<0.01$; full differential expression analysis is available in Supplementary Data 3 and 4). Finally, as our sample collection was performed immediately before or after cardiac transplantation (unlike post-mortem samples such as those used in GTex), we investigated whether gene expression programs related to oxidative stress were less perturbed than in samples collected post mortem. To do this, we compared oxidative stress gene expression as defined by genes in the GO term "Response to oxidative stress"(GO:0006979) in our samples to left-ventricular sample data in GTEx, as well as other KEGG and Reactome pathways (data obtained from the recount2 database $^{18}$ ). Our results suggested that our samples displayed comparable contractility-related gene expression but had significantly less oxidative-stress-related gene expression and less perturbation in other metabolic pathways (Supplementary Fig. 2C). Having established the quality of our data, we limited our network-based downstream analyses to the $40 \%$ genes most variably expressed between failing and nonfailing hearts $(n=$ 7960) in order to limit inflation of correlation between low covariance gene pairs.

Cardiac coexpression maps reveal dynamic network topology. We inferred undirected, disease-state-specific gene coexpression networks. Gene regulatory network inference from coexpression is a challenging problem that no single method solves adequately in all contexts. Here, we constructed control- and HF-specific networks using methods that rely on gene coexpression (Weighted Gene Co-expression Network Analysis (WGCNA) 19,20 and Pearson correlation), inverse covariance estimation (Joint Graphical LASSO (JGL) ${ }^{21}$ ), and mutual information (ARACNe ${ }^{22}$ and ZScore) (see Methods for details. All network constructions are available at https://doi.org/10.5281/zenodo.2600420). Each of these methods has specific advantages for different questions, e.g. JGL creates a sparser network with the specific intent of reducing representation of noncausal associations, whereas WGCNA relies on denser network topology to capture modules of genes with high likelihood of interaction. Since our downstream analysis required a top-down systems view of coexpression networks, and because we planned to prioritize genes based on the change in connectivity of networks between disease states, we chose to base our subsequent analyses on WGCNA-derived networks, which represent a robust tool for this purpose..$^{23,24}$

To achieve an initial understanding of topological changes between the nonfailing and failing heart networks, we compared the structure of modules in each WGCNA-derived network (dendrograms used for module finding shown in Supplementary Fig. 3). These networks displayed different structure in HF than in control: First, the number of genes unassigned to any module was much fewer in HF (13 genes were unassigned, compared to 2614 in controls). Second, while each group of genes was specifically enriched with functional annotations as revealed by enrichR ${ }^{25}$, the HF modules had more diversity of signaling and metabolic annotations (full gene module descriptions and Benjamini-Hochberg-adjusted enrichment $p$ values are shown in Supplementary Data 5 and 6).

We then manually curated modules of genes related to four key processes involved in HF (Fig. 1b: sarcomeric and contraction genes (orange), excitation-contraction (EC) coupling (red), cardiac remodeling (green), and metabolism (blue), see Supplementary Data 11). Network connectivity changed within these process-based modules between nonfailing and failing networks. Compared to the nonfailing network (gray typeface), the failing heart network (red typeface) saw a general rewiring in connectivity within and between these modules; metabolic genes gained a few specific genes such as the protein phosphatase 1 catalytic and regulatory subunits (PPP1CC, PPP1R1A, and $P P P 1 R 3 A / B / C)$ and the muscle 6-phosphofructokinase $P F K M$ in the HF network (Fig. 1b, blue). Cardiac remodeling genes that gained connectivity were MYBPC3, MYH7, RYR2, and SGCG/D; sarcomeric and contraction genes that gained connectivity were 
a

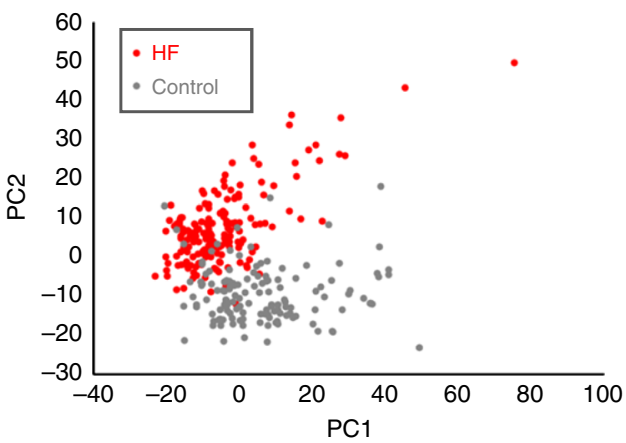

b

Sarcomeric and contraction

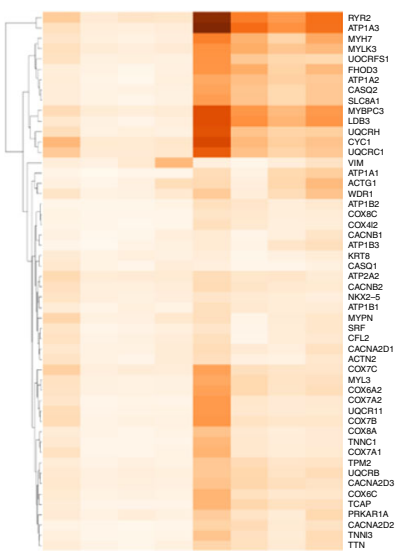

EC coupling
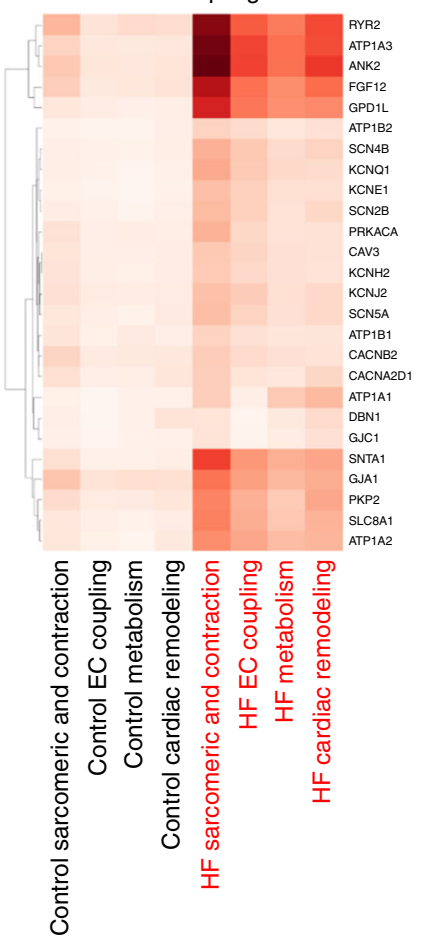

Cardiac remodeling

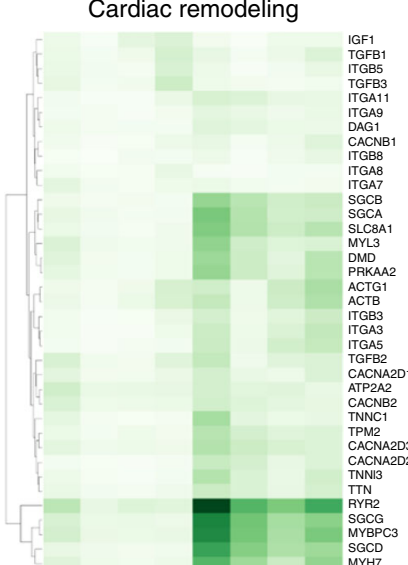

Metabolism
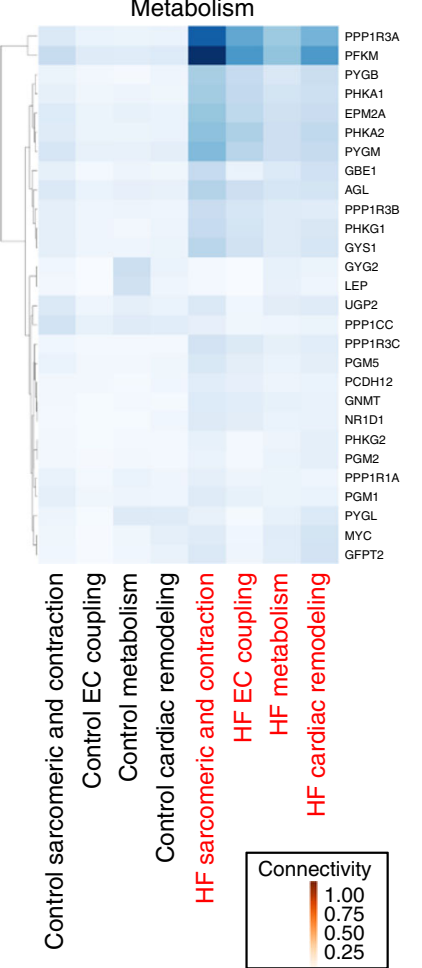

c

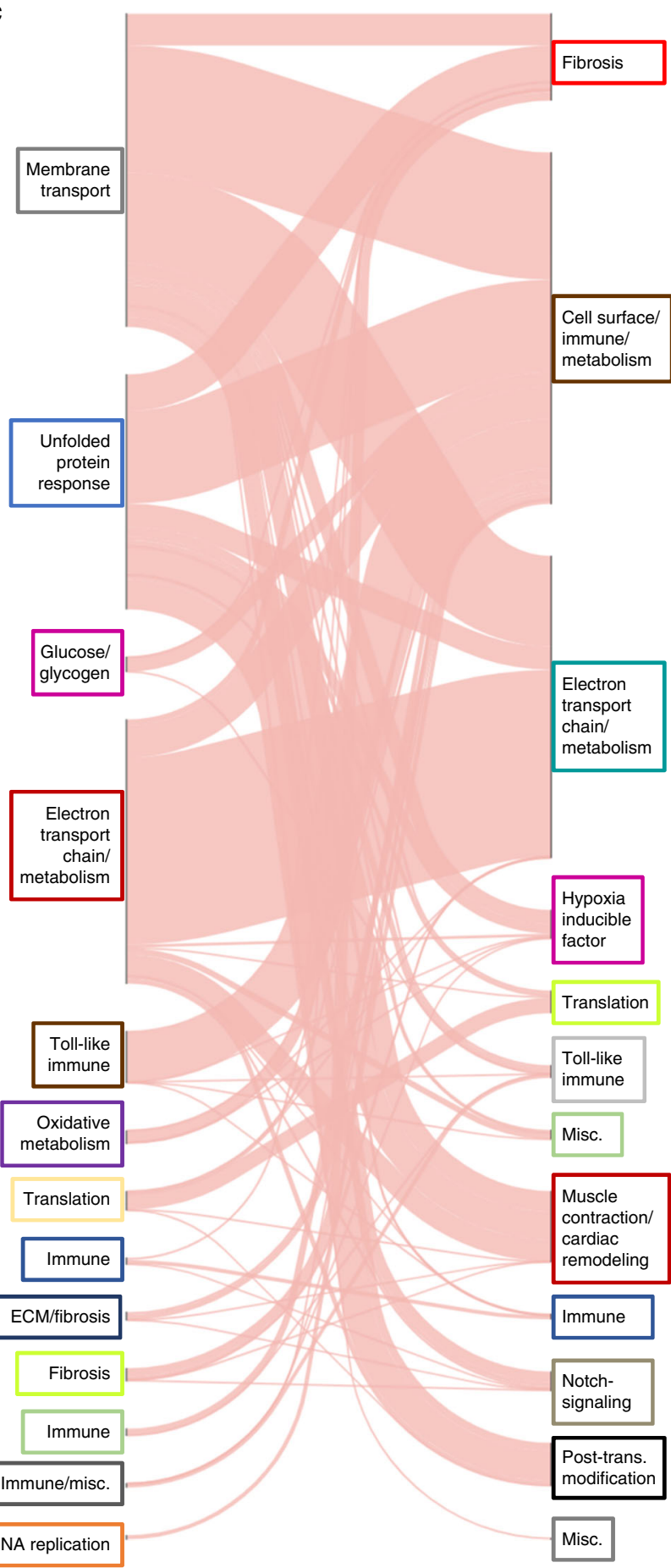

MYBPC3, MYH7, again listed due to pathway overlaps and VIM, $U Q C R H / C 1$; while for EC coupling these were $A T P 1 A 1 / 2 / 3$ and again $R Y R 2$.

Finally, plotting a Sankey diagram to observe where module membership changes from controls to HF (Fig. 1c) revealed large rewiring of coexpression structure. Shared core structure modules such as electron transport chain (ETC) and metabolism genes mostly remained in the same module (dark red in controls and turquoise in $\mathrm{HF}$ ), while the unassigned genes in the controls (gray) went mostly to the metabolism/ETC (turquoise), cell surface/immune/metabolism (brown), and fibrosis (red) modules in HF. 
Fig. 1 Regulatory rewiring of coexpression networks in HF. a Principal component analysis of gene expression profiles for 177 failing hearts and 136 nonfailing, control, hearts showing clear segregation of $\mathrm{HF}$ (red) vs. control (gray) population. b Differential connectivity of known biological processes in HF. Normalized connectivity (sum of WGCNA weights divided by maximum network weight) between representative genes from four known processes that play critical roles in HF (sarcomeric and contraction genes (orange), EC coupling (red), cardiac remodeling (green), and metabolism (blue)) to all genes from those same processes in HF and controls. Genes of each process are rows and columns are process and cohort. For example, MYBPC 3 in the cardiac remodeling process (third row in green heat map) is highly connected to sarcomeric and contraction genes and cardiac remodeling genes in the HF network (fifth and eighth columns respectively) compared to all the control processes. c River plot demonstrating changing modular assignments for genes in the HF vs. control networks. Pink lines represent individual genes, with left-sided grouping representing membership in control (left) and HF (right) network modules (indicated by color of text box) and right-sided grouping in HF network modules, with text indicating module names derived from KEGG and Reactome associations of genes within each module

High-quality tissue expression data reveal cardiac eQTLs. We then leveraged genome-wide genotypes to find gene-expressioncontrolling loci (eQTLs) in each cohort. First, we performed hidden covariate correction using the PEER package (see Methods). We used QTLtools to perform association testing for each cohort separately (see Methods) and performed a scan on the number of hidden factors to correct with PEER. ${ }^{26}$ We found that the HF cohort had more associated eQTLs than the control group (1566 vs. 936, respectively, with an overlap of 254 loci between the two groups, see https://doi.org/10.5281/zenodo.2617028 for full eQTL results); as expected these eQTLs showed proximity to known transcription factor binding sites and transcription start sites (Fig. 2a, b). We then tested these eQTLs for enrichment of regulatory associations using RegulomeDB, a database of known and predicted regulatory regions of the genome ${ }^{27}$. Here, both cohorts had several eQTLs with adjacent (within 50 base pair window) regulatory annotation (716/1566 (46\%) and 425/936 (45\%) of variants, for failing vs. control, respectively) and/or predicted for transcription factor binding (Supplementary Fig. 4A). We then compared our eQTLs with those found by the GTEx project. Our set of eQTLs contained hundreds of novel associations when compared to the GTEx database for leftventricular tissue: 831/1566 (53\% novel associations) for the HF group and 423/936 (45\% novel associations) for the control group (Fig. 2c). We also identified significant overlap with specific tissue types (e.g. artery vs. muscle) and cell types (e.g. cultured fibroblasts) (Supplementary Fig. 4B). Comparison to a recently updated version of GTEx yielded even greater overlap (Supplementary Table 2).

This percentage of newly identified eQTLs is larger than on previous, related eQTL studies ${ }^{10,28}$, and also displays greater overlap with GTEx than those found in an independent cohort of patients with dilated cardiomyopathy ${ }^{10}$. This large overlap was encouraging, and since we control for known and hidden covariates, our difference in eQTLs compared to related studies may reflect the immediate tissue collection techniques we used, and the difference in disease status (at least one third of cases in this study had ischemic disease, which was not true for comparison studies, which focused on nonischemic dilated cardiomyopathy ${ }^{10}$ ).

To assess the physiological impact of our eQTLs, we checked for overlap of our eQTL associations with existing variants in the GWAS catalog 29 . First, we did a simple, direct SNP overlap check with the GWAS variants (GWAS catalog variants with an LD cutoff of 0.6, using $\mathrm{SNiPA}^{30}$ to check for LD overlaps). For HF eQTLs, this revealed 41 variants, among which we found 25 associations with sudden cardiac arrest, heart rate variability, and coronary heart disease among other diseases/traits, whereas 33 of the nonfailing control eQTLs had associations in the catalog, including QT interval and heart rate variability traits (Supplementary Data 7 and 8). To assess whether the magnitude of this overlap was higher than expected, we used SNPSNAP ${ }^{31}$ to generate two sets of 10,000 random variants each with the same
LD and gene density characteristics as our failing and nonfailing eQTLs. The average overlap of these random sets to GWAS variants was 0.1 for both sets, yielding empirical $p$ values of less than 0.001 in each set and confirming that our overlap is higher than expected.

To expand our analysis of overlap of our eQTL findings with GWAS, we used eCAVIAR ${ }^{32}$, a high-resolution method that leverages SNP density to perform colocalization enrichment tests between hits in a high-powered, publicly available coronary artery disease GWAS and nearby eQTLs from our analysis (see Methods). We chose this coronary artery disease GWAS for comparison not only because it is one of few related to causes of HF that are appropriately powered for this high-resolution method, but believed it to be a reasonable disease surrogate for comparison based on the $36 \%$ of explanted failing hearts in our study that had undergone coronary artery bypass grafting (Supplementary Table 1). This method found seven regions nearby seven genes that have coronary artery disease-associated SNPs and that are significantly colocalized with our eQTLs (see Methods).

We then interrogated which gene modules uncovered by WGCNA were controlled by eQTL loci in concert by examining the fraction of genes that were e-genes of in the eQTL analysis (Fig. 2c). The modules with the top fraction of e-genes was turquoise in $\mathrm{HF}$ and dark red in the controls $(40 \%$ and $32 \%$, respectively), both of which correspond to ETC and metabolism genes. In HF, the next modules with the most fraction of e-genes were the brown module, a combination of cell surface, immune, and metabolism genes, as well as the dark red module, comprised of muscle contraction and cardiac remodeling genes. For the control cohort, the next module most enriched with e-genes was the gray/unassigned module, indicating a less cohesive regulatory structure; then followed by the blue module dominated by unfolded protein response genes.

We went on to identify modules of coordinating genetic loci and associated networks of genes within these associations by finding nontrivial connected components (i.e. with more than three nodes) within the bipartite association graph of variants and genes, including WGCNA edges with weights larger than the median (Fig. 2d). Notably, we found two eQTLs within a region enriched with predicted histone modifications that controlled a network of several TAS2R members in-cis, a family of G-proteincoupled receptors, in both failing and control groups (Fig. 2e, f, r10492099 and rs4763223). As TAS2R receptors can have high homology in some regions, we checked for potential probe crosshybridization. We compared sequences for all TAS2R gene probes for the GeneChip ST1.1 array by BLASTing them against human transcript sequences (evalue cutoff of 0.01 , with at least $12 / 25$ exact matches). The genes TAS2R43-TAS2R46 had overlapping, high similarity matching probes, suggesting possible crosshybridization, while the rest of the receptors' probes were deemed independent by this analysis. These associations, prevalent in both cohorts, highlight a common module of 

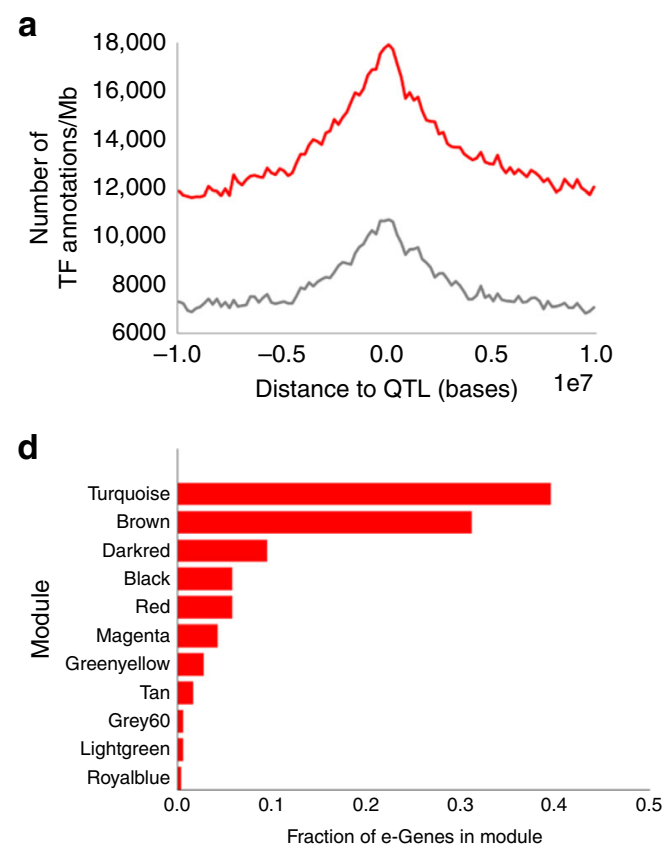

e

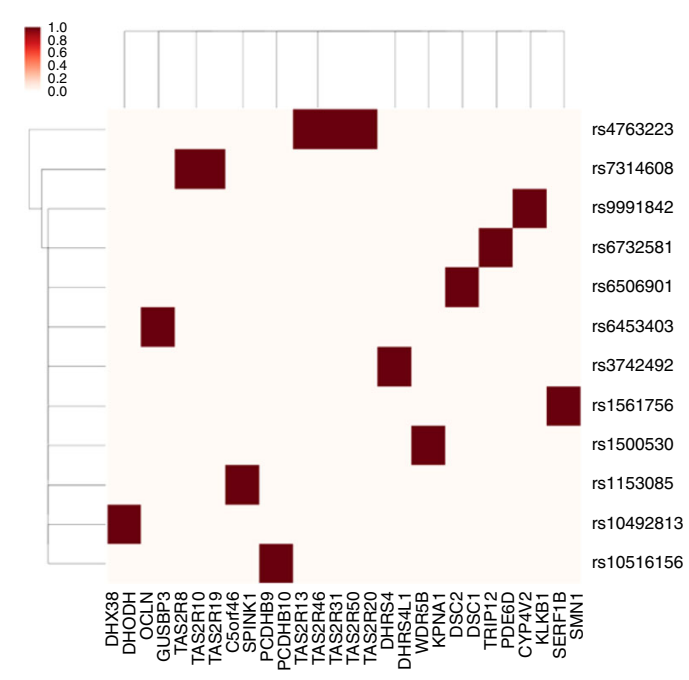

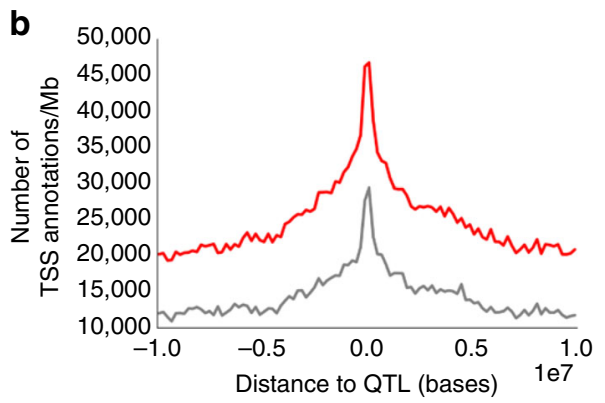
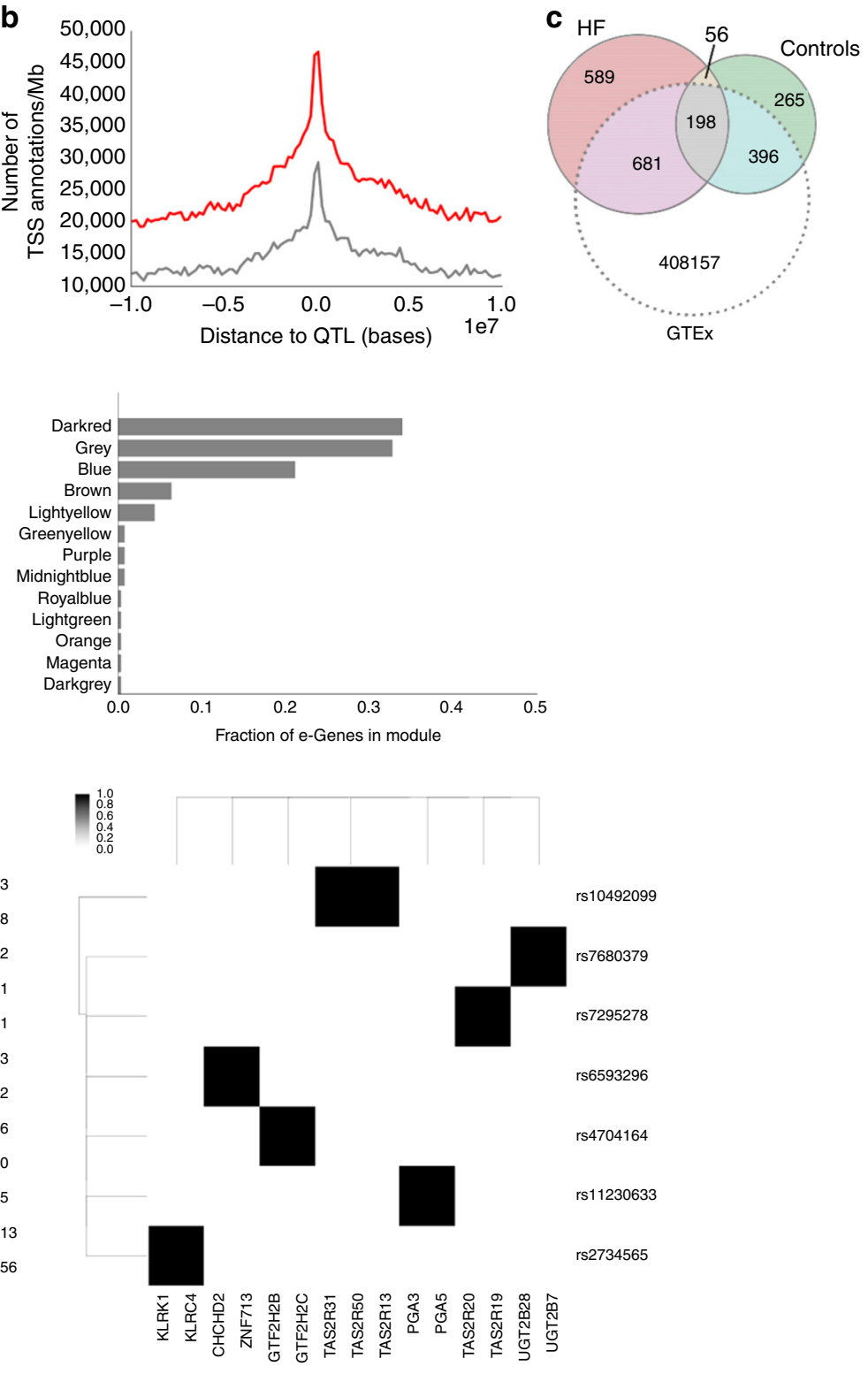

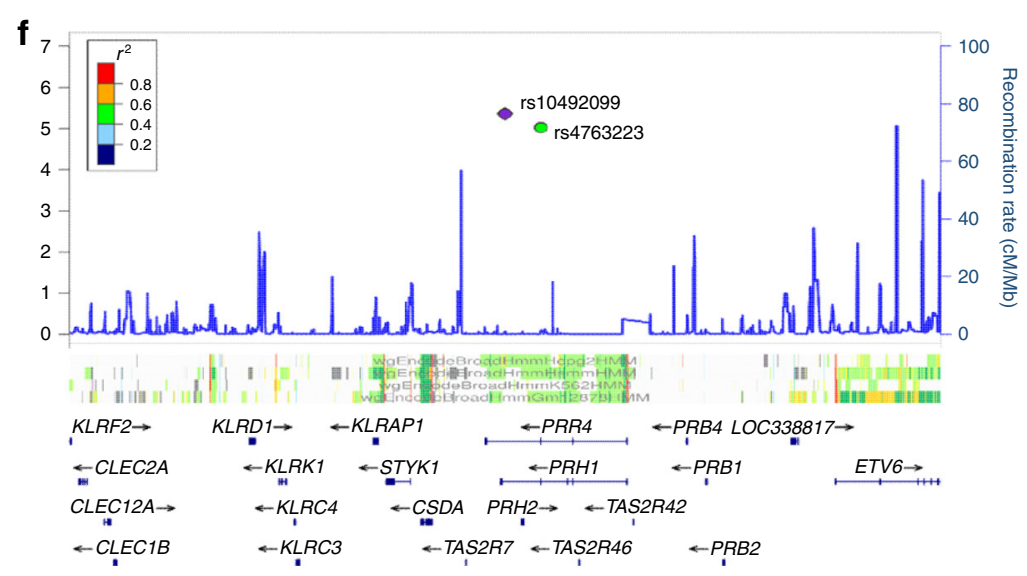

Fig. 2 High-quality tissue expression reveals previously undetected cardiac eQTL associations. Transcription factor (a) and transcription start site (b) annotation to eQTL distance distributions for failing (red) and control hearts. c Number of cis eQTLs found for each group that overlapped with GTEx eQTLs. d Fraction of modules with genes found to be controlled by at least one eQTL in HF (red, left) and controls (gray, right). e Heat map indicators for variants controlling multiple genes in-cis in HF (red, left) and controls (gray, right). In the rows are SNPs controlling genes (columns) colored intensely if the SNP controls the gene. $\mathbf{f}$ Variants from one locus control a network of G-protein-coupled receptors TAS2R present in both the failing and control groups 
G-protein-coupled receptors that have been previously observed to be expressed in the healthy and failing heart ${ }^{33,34}$, and that may play a role in the regulation of arrhythmia and contractility ${ }^{35}$.

In summary, our heart transplant cardiac samples and inferred gene coexpression networks enabled us to find several previously unidentified cardiac eQTLs in the failing and nonfailing heart. Many of the eQTL variants were also associated with cardiac phenotypes in GWAS and some are associated with genes in highly connected parts of the coexpression network, suggesting coordinated regulation.

Dynamic network topology illuminates central HF regulators. Next, we used our network topology to identify and prioritize genes that were dynamically connected between the failing and control heart networks. Our goal was to identify genes whose network connectivity was increased in the disease state (i.e. HF), but specifically to pathways known to be relevant to the global control of HF mechanisms. We achieved this by ranking genes on two connectivity metrics: (i) differential local network connectivity for each gene between control and failing networks, and (ii) change in each gene's connectivity globally to HF-relevant molecular pathways manually curated from KEGG and Reactome (curated list of pathways included in Supplementary Data 9). We defined local connectivity (LC) as the change in number of edges for each gene between the control and HF networks. Global connectivity (GC) was defined as the number of curated HFrelevant pathways to which each gene was significantly differentially connected in the control vs. HF networks, taking into account network distance (see Methods, Supplementary Fig. 5).

Using GC and LC, we assigned each gene to one of four categories (Fig. 3a): Noncoordinators were genes with decreased LC and GC between the control and HF networks, making them less likely to be highly impactful in the disease state. Local coordinators had significant increases in LC but decreased GC, indicating high coexpression, but mostly with genes unrelated to global HF processes. Pathway coordinators were genes with increased GC but overall low LC, indicating an increased association with global HF processes, but overall low impact with respect to gains in coexpression. Finally, central coordinators increased both in GC and LC, and therefore represent genes with increased local coexpression involving an increased number of global HF-relevant processes (a list of genes and assigned status are included in Supplementary Data 10).

After classifying each gene in this way, we focused on central coordinators (Fig. 3b, top right) in order to capture those genes with the most dynamic connectivity in disease. In addition to increased connectivity with HF-relevant pathways in the HF vs. control networks, these central coordinators were enriched in OMIM/KEGG cardiomyopathy terms and pathways (hypertrophic and dilated cardiomyopathy KEGG pathway and OMIM terms, Fisher exact test $p$ values $<0.001)$. This includes the myosin binding protein C3 (MYBPC3) which has previously been implicated in the Mendelian cardiac muscle diseases, hypertrophic cardiomyopathy and dilated cardiomyopathy ${ }^{36,37}$ (Fig. 3b). Particularly, we noted that genes with highest increases in global connectivity regulated HF-related pathways across several cardiomyocyte-relevant processes including metabolism, muscle contraction, and cardiomyopathy-related genes (Fig. 3c). These data are in concordance with transcriptomic data from murine myocardium with and without exposure to transaortic constriction, which revealed prevalent gene modules associated with mitochondrial and cytoskeletal gene ontologies. ${ }^{28}$ In contrast, prioritization by differential gene expression between failing and control myocardium did not reveal many genes genetically associated with known cardiovascular disease pathways. We found no relationship between differential expression and either global or local differential connectivity. Only 1 of the top 20 highly differentially expressed genes was associated with cardiovascular disease pathways in KEGG or Reactome compared to 5 out of the top 20 for the connectivityderived list-a significant enrichment difference ((Fisher exact test $p$ value $<0.001)$, Supplementary Fig. 2).

Among those central coordinators whose network connectivity was maximally changed in the failing heart was protein phosphatase 1 regulatory subunit $3 \mathrm{~A}(P P P 1 R 3 A)$, with one of the highest changes in GC between control and failing hearts (green typeface, Fig. 3b). $P P P 1 R 3 A$, which encodes a musclespecific regulatory subunit of protein phosphatase $1(\mathrm{PP} 1)^{38}$, has not been previously associated with HF. To examine the importance of $P P P 1 R 3 A$ to cardiomyocyte hypertrophy across cardiomyopathic etiologies, we also examined its importance in a cardiomyopathy pathway (hypertrophic cardiomyopathy, KEGG), and found that its differential connectivity to this pathway (Fig. 3d) exceeded even that of MYH7 (Fig. 3e), an exemplar cardiomyopathy gene. Additionally, we noted that connectivity of $P P P 1 R 3 A$ to our lists of sarcomeric and contraction genes was increased significantly in HF (Fig. 2b). Previous work indicates $P P P 1 R 3 A$ contains a glycogen-binding domain $^{39}$ and is thought to promote skeletal muscle glycogen synthesis, and variants in $P P P 1 R 3 A$ have been associated with decreased insulin sensitivity ${ }^{40,41}$. Our own networks showed increased connectivity of $P P P 1 R 3 A$ to metabolic pathways in control and failing myocardium as well (Figs. 1b, 3c). As cardiac metabolism in HF is known to switch toward a glucose-based metabolism, and as metabolic pathways were significantly connected to PPP1R3A in our HF network (Fig. 3c), we hypothesized that this gene would play an important role in the transition from healthy to failing myocardium.

PPP1R3A knockdown ablates HF phenotypes in vitro. To investigate the role of $P P P 1 R 3 A$ in $\mathrm{HF}$, we first determined the effect of perturbing $P P P 1 R 3 A$ expression via RNA silencing on global gene expression in vitro. We used RNA sequencing to measure global gene expression at various time points with and without PPP1R3A knockdown in phenylephrine-treated NRVMs (an in vitro model of cardiomyocyte hypertrophy and HF signaling, Fig. 4a, Supplementary Fig. 6). Global expression of central coordinators significantly changed after knockdown and/or phenylephrine treatment at both time points ( ${ }^{*}$ indicates $\mathrm{FDR}<$ 0.05, Fig. 4b). The expression of central coordinators (Fig. 3a, b) was highly altered by knockdown of PPP1R3A in NRVM both with and without a hypertrophic stimulus (phenylephrine, Fig. 4b). Genes whose change in expression met statistical significance in the global RNA-sequencing dataset were overrepresented among these highly dynamic central coordinators ( ${ }^{*}$ indicates FDR 0.05 , Fig. 4b), providing evidence that PPP1R3A is a central regulator of this network, as manipulation of its expression directly causes significant network perturbation. Consistent with these findings, knockdown of $P P P 1 R 3 A$ also protected NRVMs against cellular hypertrophy caused by phenylephrine both by measures of cell size and the ratio of Myh7/ Myh6 gene expression over time (Fig. 4c). Taken together, these results indicate that reduction of $P P P 1 R 3 A$ expression slows cellular HF pathology and its associated signaling in vitro by acting as a central regulator in the network of hypertrophy- and HF-relevant pathways.

Noting that many of the central coordinators significantly regulated by $P P P 1 R 3 A$ knockdown were metabolic genes (e.g. GLUT4, PFK, multiple subunits of NADH ubiquinone oxidoreductase and COQ10, Fig. 4b) and that prior work has implicated 
a

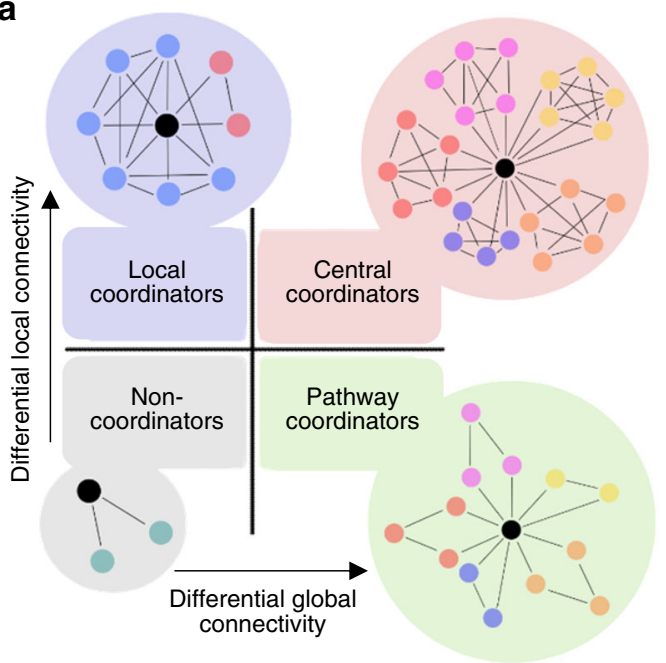

c

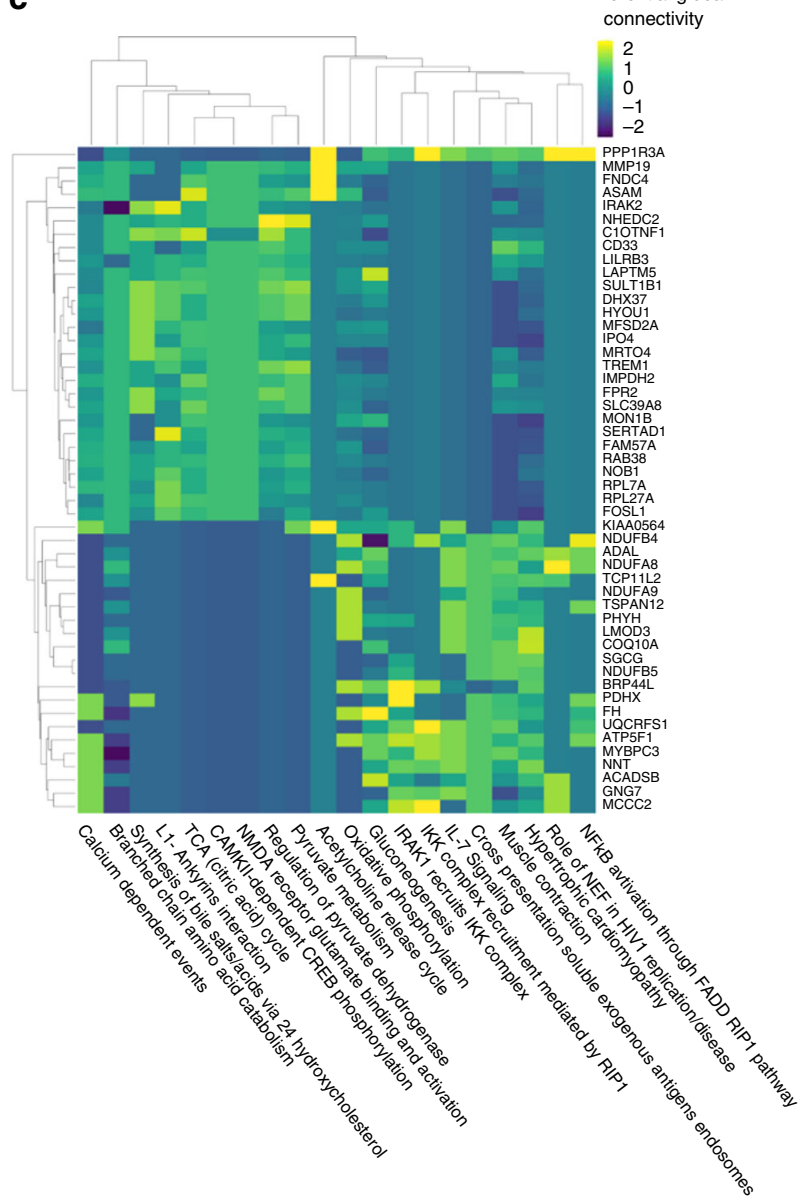

b

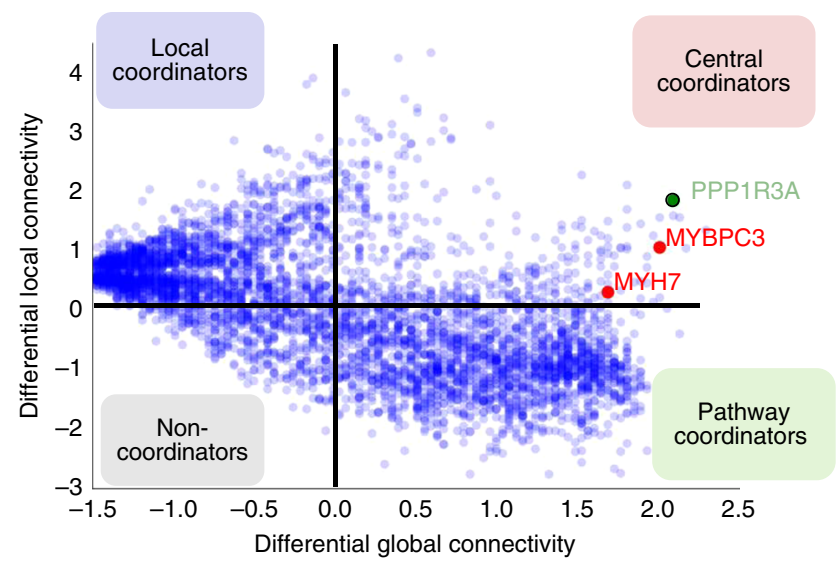

d MYH7 differential HCM pathway connectivity

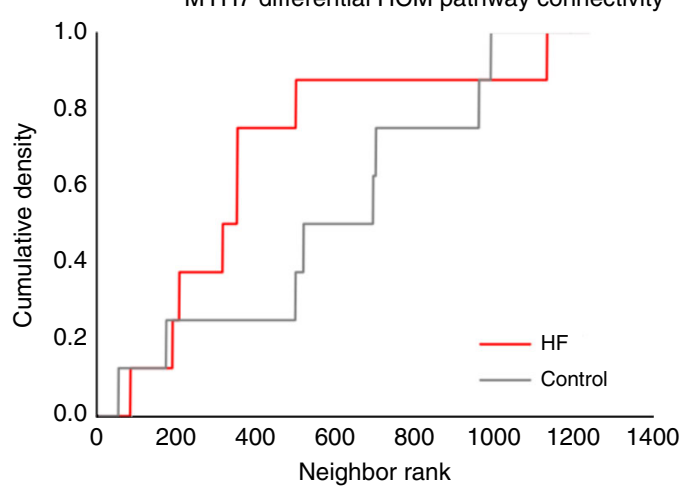

e PPP1R3A differential HCM pathway connectivity

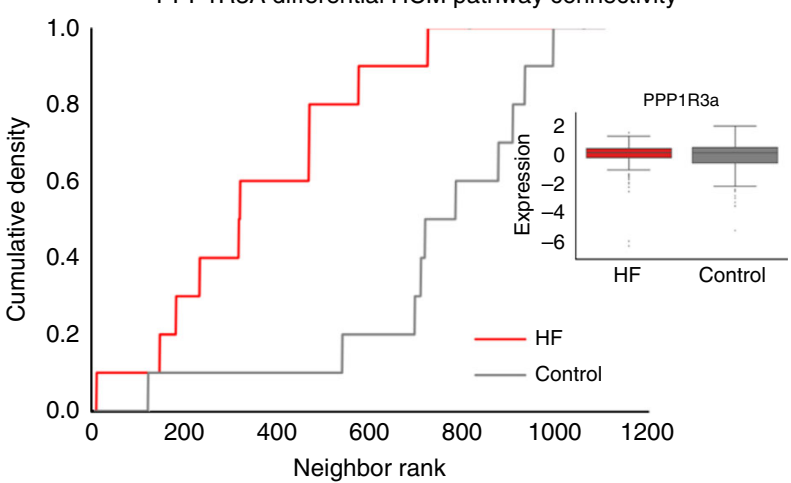

Fig. 3 Gene prioritization through network topology. a Diagrammatic representation of roles of local and global connectivity in defining each gene's coordinator status. Local connectivity (LC) is the per-gene change in coexpression edges in the HF vs. control network. Global connectivity (GC) represents the enrichment of HF-relevant pathways in a gene's neighborhood between HF and control networks (see Methods). b Change in local and global connectivity for all genes between control and HF networks identified PPP1R3A (green font) as a central coordinator in HF, indicating its increased association with HF-relevant pathways as well as coexpression relationships in the HF vs. control networks. c Gene-pathway (rows-columns)

differential connectivity matrix for genes ranked highest for global network connectivity that is differentially increased between non-heart-failure and heartfailure conditions. Differential connectivity is measured by the KS statistic between the distribution of ranks for pathway genes in $\mathrm{HF}$ and non-heart-failure conditions. In particular, metabolism, HF and cardiomyopathy pathways (yellow indicates an increase in connectivity to the given pathway (columns) in HF compared to control samples). d Difference in cumulative membership distributions for the KEGG Hypertrophic Cardiomyopathy (HCM) pathway for the myosin gene MYH7 which is known to be involved in HCM. e Difference for HCM pathway enrichment in the protein phosphatase 1 regulatory subunit PPP1R3A is more dramatic than for MYH7. Inset: PPP1R3A transcriptional expression in HF and Control cohorts is unchanged 
a

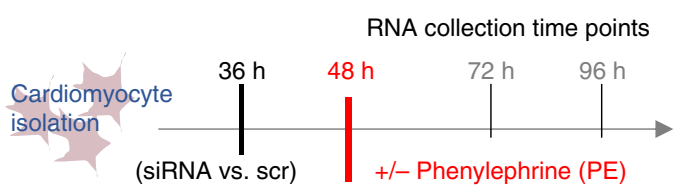

b

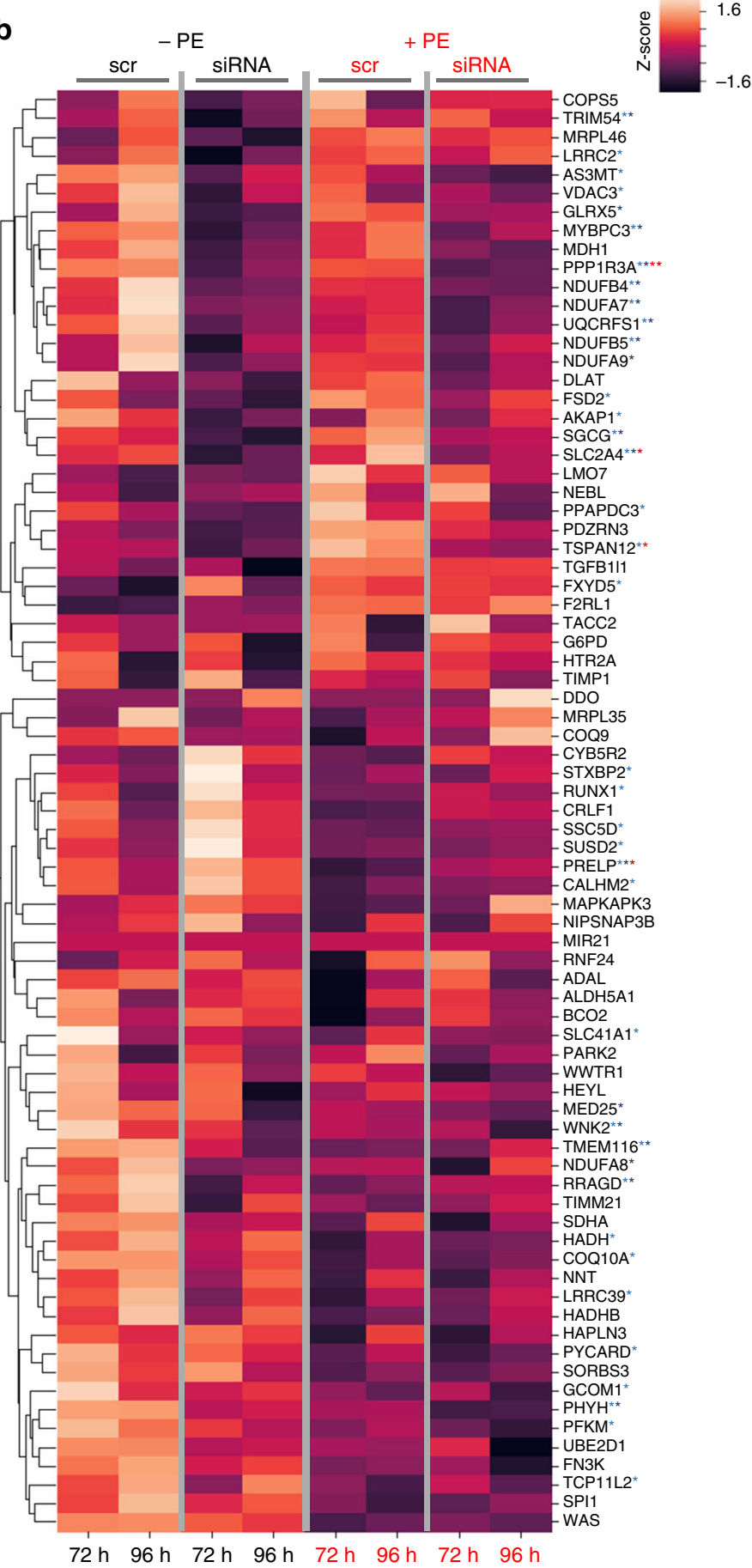

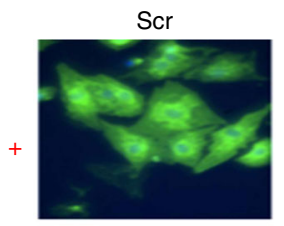

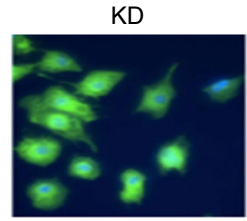

남
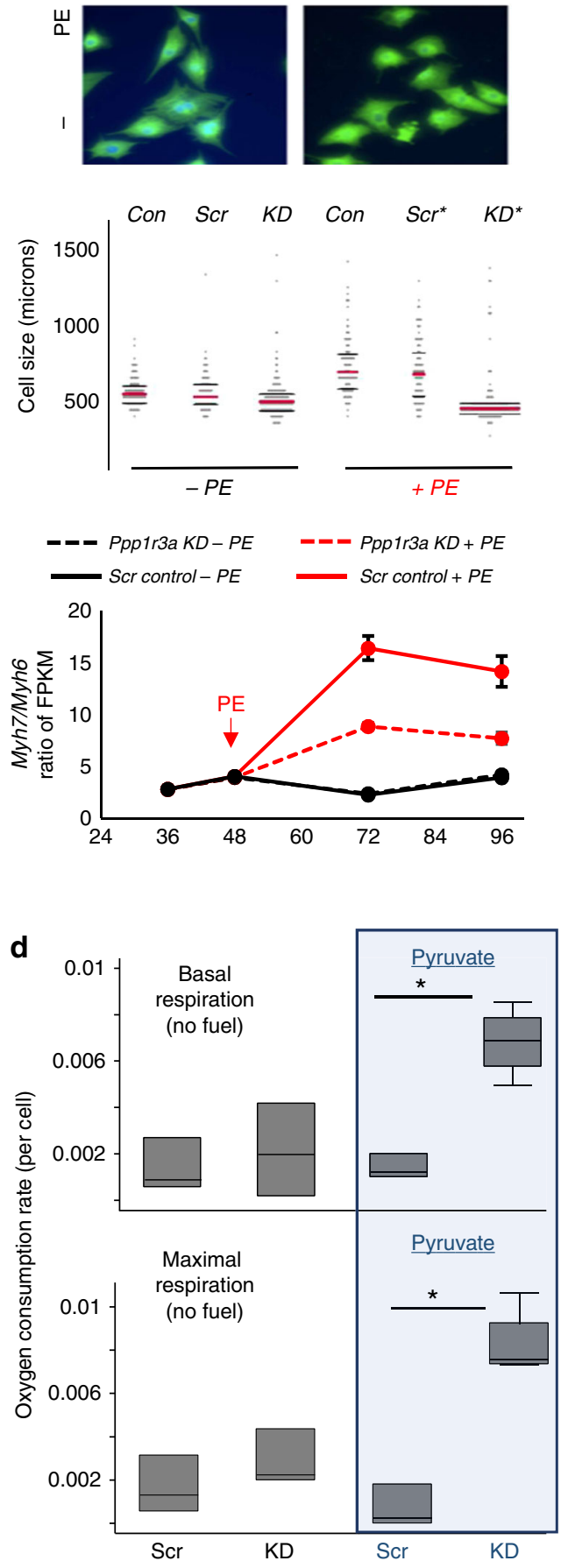

$P P P 1 R 3 A$ in striated muscle glycogen metabolism, we hypothesized that PPP1R3A's role in the development of HF was related to the metabolic switch from respiratory to glycolytic glucose metabolism observed in failing myocardium. We found that under phenylephrine-treated conditions, PPP1R3A knockdown resulted in the alteration of genes critical to glucose metabolism such as glucose transporters GLUT1 and GLUT4 (upregulation and downregulation, respectively, Supplementary Fig. 6). Further, under normal NRVM culture conditions, knockdown of PPP1R3A induced a significant downregulation of critical regulators of oxidative metabolism, such as the pyruvate dehydrogenases $P D K 2$, the carnitine palmitoyltransferase $C P T 1 B$ (Supplementary Fig. 7). Pyruvate dehydrogenase kinases (e.g. $P D K 2)$ are major molecular drivers of decreased respiratory 
Fig. 4 PPP1R3A knockdown in vitro reveals metabolic regulation in cardiomyocytes. a Experimental design. NRVMs were isolated, transfected with siRNA $36 \mathrm{~h}$ later. Phenylephrine or vehicle treatment started at $48 \mathrm{~h}$. RNA was collected at 36, 48, 72 and $96 \mathrm{~h}$ after isolation. b Clustered heat map of NRVM transcriptional expression of central coordinators in response to PPP1R3A knockdown (measured by RNAseq). Expression is shown from NRVMs at 72 and $96 \mathrm{~h}$ after isolation normalized to pretreatment expression, and displayed as per-gene $z$-scores. Data from cells with and without phenylephrine (PE) are shown on the left and right sides of the heat map with and without siRNA knockdown as indicated. Stars indicate central coordinators significantly differentially regulated by PPP1R3A knockdown (FDR $<0.05$, red stars indicate significance in the PE-treated group (red at $72 \mathrm{~h}$, dark red at $96 \mathrm{~h}$ ) and blue stars indicate significance in the untreated group after PPP1R3A knockdown at 72 (light blue) and 96 (dark blue) hours). c PPP1R3A knockdown protects against hypertrophic stimulus of phenylephrine treatment. Upper panel: Cell size measurements of a sample of cells under phenylephrine and normal conditions, with and without PPP1R3A KD reveal reduced hypertrophy in NRVMs treated with PE and PPP1R3A KD compared to PE-treated cells with and without scramble siRNA transfection ( $p<1 \mathrm{e} 10^{-4}$ (ANOVA), ${ }^{*}=p<1 \mathrm{e} 10^{-3}$ by Bonferroni post test. $n=100$ cells for each group, red bars indicate mean, black bars indicate one standard deviation). Lower panel: Myh7/Myh6 ratio, a marker for HF, is decreased in PPP1R3A KD NRVMs treated with PE compared to those transfected with scrambled siRNA control at 72 and $96 \mathrm{~h}$ after isolation ( $p<1 \mathrm{e} 10^{-2}$ for both comparisons, error bars represent $95 \%$ confidence intervals). d Respiratory pyruvate metabolism increases after PPP1R3A knockdown. Knockdown of PPP1R3A leads to increased basal and maximal respiratory metabolism of pyruvate as measured by oxygen consumption in NRVM (basal respiration: $p=0.02$, maximal respiration: $p=0.005$, center line indicates median, box indicates IQR, and whiskers indicate next adjacent value. $n=3$ biologically independent samples for the siRNA/pyruvate group and $n=4$ for all other groups). Source data for this figure are provided in a source data file

glucose metabolism in HF via inactivation of pyruvate dehydrogenase ${ }^{42}$. This results in shunting of pyruvate away from the oxidative TCA cycle into lactate, thus decreasing energetic efficiency of glucose metabolism in cardiomyocytes. We therefore hypothesized that decreasing $P P P 1 R 3 A$ expression would lead to liberation of respiratory metabolism, and found that siRNAmediated knockdown of PPP1R3A leads to increased basal and maximal respiratory metabolism of pyruvate by NRVM as measured by oxygen consumption ( $p=0.02$ (basal respiration) and $p<0.01$ (maximal respiration) Fig. $4 \mathrm{~d}$, blue boxes, see Methods).

Ppp1r3a ${ }^{-1-}$ mice are protected against $\mathrm{LV}$ dysfunction. We then investigated the effect of PPP1R3A on HF in vivo using a model of pressure overload, transaortic constriction (TAC). Compared to Ppp1r3a $a^{+/+}$, Ppp1r3a-1- mice were protected from TAC-induced left-ventricular ( $\mathrm{LV})$ dysfunction as measured by fractional shortening ( $p=0.002$ (ANOVA), Fig. 5a). This effect was associated with unchanged levels of the HF markers Nppa and $N p p b$ in the LV of Ppp1r3a-1- TAC vs. sham mice compared to the expected TAC-induced increase observed in $P p p 1 r 3 a^{+/+}$mice (Fig. 5b, $N p p a: p=0.006$ (ANOVA), $N p p b: p=0.008$ (ANOVA)). No significant difference was found across groups in the ratio of $M y h 7 / M y h 6$ expression (Fig. 5b, $p=0.11$, ANOVA).

In accordance with these findings, there was no difference between Ppp1r3a-/- TAC and sham in cardiomyocyte size, while Ppp1r3a $a^{+I+}$ animals showed expected cellular hypertrophy after TAC (Fig. 5c ( $p=0.004$ (ANOVA)). After TAC, heart weight/body weight ratio varied significantly between groups, though TAC comparisons within genotype did not reach statistical significance by Bonferroni post test $\left(p=0.02\right.$, ANOVA, Ppp1r3a $a^{+/+}$TAC vs. Sham $p=0.07$ Bonferroni post test, Supplementary Fig. 8). LV fibrosis was also unchanged in Ppp1r3a-l- animals after TAC, as opposed to the expected increase in fibrosis seen in $P p p 1 r 3 a^{+/+}$ animals in response to TAC (Fig. $5 \mathrm{~d}, p<0.0001$ (ANOVA)).

\section{Discussion}

We have constructed a comprehensive gene regulatory map of human heart failure (HF). This effort has been facilitated by a systematic approach to the collection of control and failing heart tissue from the operating rooms of cardiac transplant centers and the resulting measurements have allowed us to describe several previously unrecognized molecular features of HF. Notably, the network structure of HF differs markedly from that of nonfailing heart tissue. Specifically, we find that the control network has a large number of genes (2614) not associated with modules, whereas in the HF network, only 13 genes remained unassociated, providing evidence for increased connectivity in the HF network. Further, in the HF network, there is significant rewiring of genes to new processes (Fig. 1c), and an array of changes in coexpression relationships of central genes to key processes such as sarcomeric structure, excitation-contraction coupling, metabolism, and cardiac remodeling.

The inferred networks also aided in the discovery of new eQTLs in the nonfailing and failing contexts. Notably, we found a greater number of eQTLs in the failing heart, half of which were not previously reported, but that were still implicated in higher phenotype associations in GWAS. In some cases, the expression of local subnetworks of genes were found to be associated with one locus, such as several members of the TAS2R G-proteincoupled receptor family, receptors typically associated with the sensation of taste but recently found to be variously expressed in cardiac tissue ${ }^{33}$. Further, these eQTLs were enriched for regulatory annotations, which were more prevalent in the failing heart cohort. Thus, these newly identified eQTLs are not only important for identifying potential regulatory DNA, but also novel molecular actors in HF that would not have been discovered in healthy tissue alone.

In addition to identified eQTLs, comparison of coexpression structure between disease and control networks highlighted genes whose connectivity changed meaningfully between the two cohorts, regardless of change in mean expression. This reveals central coordinating genes in HF that would otherwise be missed by examination of individual gene expression alone. This phenomenon has been observed across human diseases, ${ }^{43,44}$ and highlights a critical feature of coexpression networks: they capture the global complexity of regulation beyond individual changes in expression to identify genes pivotal in disease. Here, our strategy classified genes both by their local connectivity and their network distance to HF-relevant pathways (global connectivity) to identify $P P P 1 R 3 A$ as a gene with a putative role in HF. PPP1R3A would not have been identified without this network approach, given that its own expression is not altered drastically in disease. Subsequent molecular investigations demonstrated its effect on other central coordinators between the control and HF networks as well as a deleterious effect on contractile function in the setting of pressure overload in vivo. Although this gene has not previously been associated with human cardiac disease, studies in both mouse and human have found that loss-of-function mutations in PPP1R3A manipulate metabolic pathways in skeletal muscle, and our own analysis implicated it in pyruvate, and other metabolic pathways (Fig. $3 \mathrm{c}$ and Fig. S7) ${ }^{41,45}$. Elimination of PPP1R3A in a murine model of cardiomyopathy revealed a maladaptive role for 
a

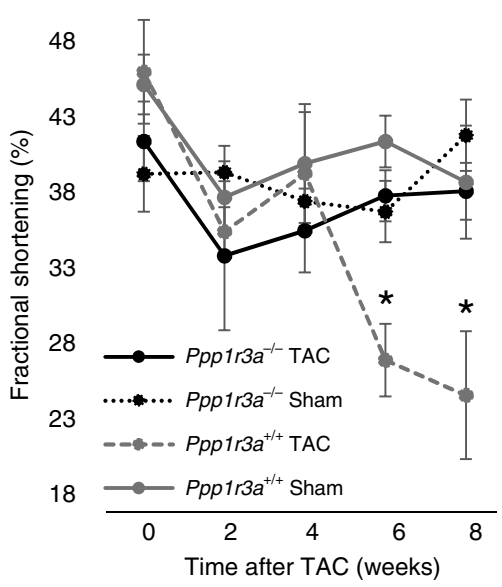

C
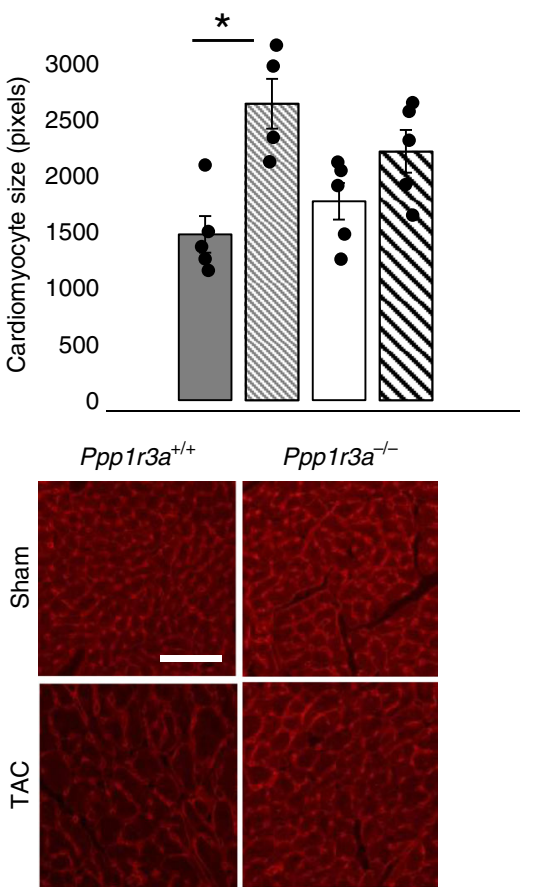

b

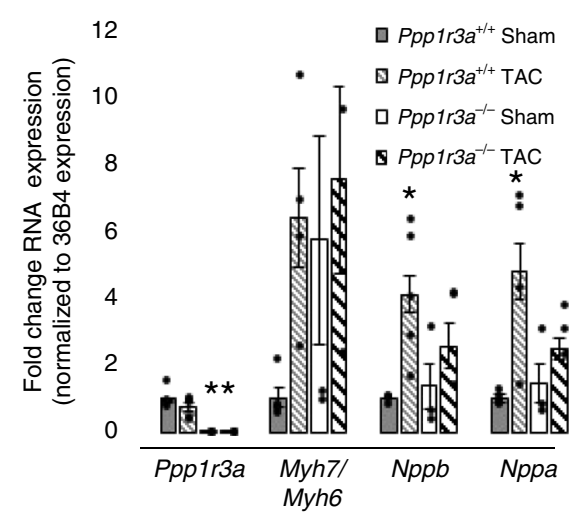

d
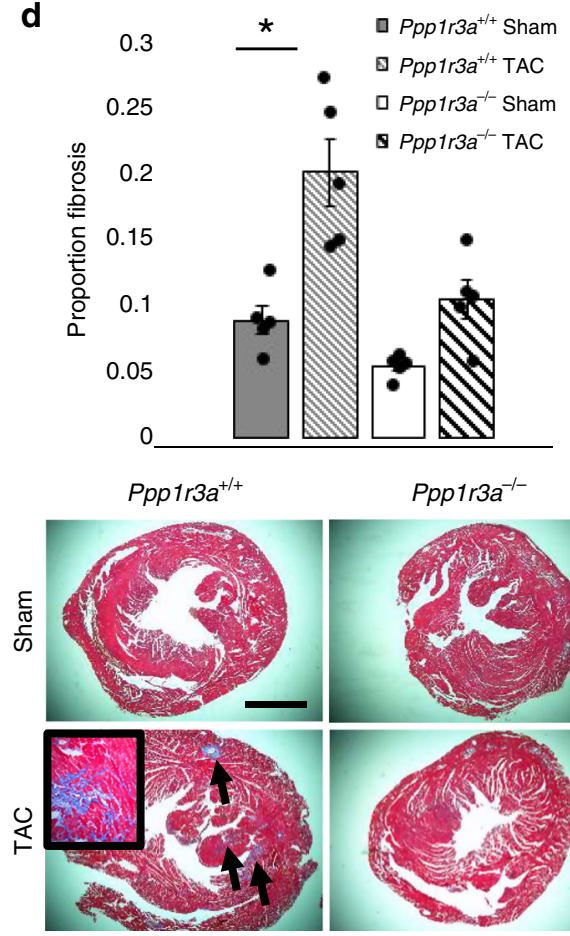

Ppp1r3a ${ }^{-/-}$

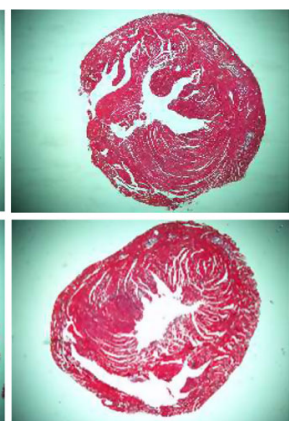

Fig. 5 Cardiac effects of PPP1R3A ablation in vivo. a Fractional shortening is preserved in Ppp1r3a- - mice after TAC. At 6 weeks $(n=5$ animals for all groups), Ppp1r3a $a^{-/}$TAC $37.6 \pm 4.3 \%$, Ppp1r3a $a^{-/}$Sham $36.5 \pm 4.7 \%$, Ppp1r3a $a^{+/+}$TAC $26.7 \pm 9.5 \%$, Ppp $1 r 3 a^{+/+}$Sham 41.2 $\pm 8.4 \%, p=0.03$ ANOVA and $p=0.03$ for Ppp1r3a-/- TAC vs. Sham by Bonferroni post test. At 8 weeks ( $n=5$ for all groups), Ppp1r3a $/-$ TAC $37.9 \pm 2.8 \%, P p p 1 r 3 a^{-/}-$Sham $41.6 \pm$ 6.8\%, Ppp1r3a+/+ TAC 24.4 $\pm 9.0 \%$, Ppp1r3a+/+ Sham $38.5 \pm 2.8 \%, p=0.002$ (ANOVA) and $p=0.01$ for Ppp1r3a $/-$ TAC vs. Sham (Bonferroni post test). $\mathbf{b}$ Gene expression of HF markers is not increased in Ppp1r3a-/- animals after TAC: PPP1R3a: $p<0.0001$ (ANOVA), $p<0.0001$ Ppp1r3a $+/+$ vs Ppp1r3a $a^{-/}$TAC and Ppp1r3a+/+ vs. Ppp1r3a-/- Sham, but $p=0.41$ Ppp1r3a+/+ TAC vs. Sham (Bonferroni post test). MYH7/MYH6: $p=0.11$ (ANOVA), $p=0.45$ Ppp1r3a+/+ TAC vs. Sham and $p=1.0$ Ppp1r3a-/- TAC vs. Sham (Bonferroni post test). Nppa: Ppp1r3a+/+ Sham $1 \pm 0.18$ (mean fold change \pm SD), Ppp1r3a+/+ TAC: $4.8 \pm 2.6$, Ppp1r3a-/- Sham: $1.5 \pm 1.1$, Ppp1r3a $/-$ TAC: $2.5 \pm 1.1 . p=0.006$ (ANOVA), $p=0.006$ Ppp1r3a $a^{+/+}$TAC vs. Sham and $p=1.0$

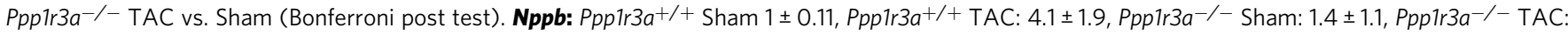
$2.6 \pm 1.5 . p=0.008$ (ANOVA), $p=0.01$ Ppp1r3a+/+ TAC vs. Sham and $p=1.0$ Ppp1r3a $/-$ TAC vs. Sham (Bonferroni post test). c Preservation of cardiomyocyte size in Ppp1r3a-/- animals after TAC: $p=0.004$ (ANOVA), $p=0.004$ Ppp1r3a $a^{+/+}$TAC vs. Sham and $p=0.64$ Ppp1r3a $-/-$ TAC vs. Sham (Bonferroni post test). Scale bar indicates $20 \mu \mathrm{m}$ length. d Fibrosis is not increased in Ppp1r3a-/- animals after TAC ( $p<0.0001$ (ANOVA), $p=0.001$ Ppp1r3a+/+ TAC vs. Sham and $p=0.22$ Ppp1r3a-/- TAC vs. Sham (Bonferroni post test). Scale bar indicates $200 \mu \mathrm{m}$ length. ${ }^{\star} p \leq 0.05$ (Bonferroni post test), TAC transaortic constriction. Error bars indicate standard error of the mean. Source data for this figure are provided in a source data file

this gene in HF, and our in vitro studies highlight the metabolic switch of failing myocardium: toward inefficient glycolytic glucose metabolism and away from the use of pyruvate in respiratory metabolism (Fig. 4d).

While a great strength of this study is its immediate isolation of RNA from freshly explanted human tissue, the resultant networks are not based on gene expression from a single cell type, but rather whole cardiac tissue. While the expression-based networks we use lend themselves to the construction of networks that bridge cell types, we cannot state with certainty that the identified central coordinators are resultant of gene-gene interactions within cardiomyocytes alone, though many of them changed 
significantly with Ppp1r3a knockdown in NRVMs (Fig. 4a). In the same vein, as the causes of HF leading to transplant are diverse, the network-based hypotheses generated by this work are likely to highlight final common pathways of HF resulting from diverse etiologies. This can be viewed as a strength of this work as it is applicable across these multiple etiologies; however, additional studies investigating the early stages of specific HF etiologies will add equally to the literature in future. It must also be noted that, due to the nature of the cohort of control hearts available for transplant, control hearts here are not free of disease (e.g. diabetes, Supplementary Table 1). Though there are more male hearts included in the HF group than control hearts, principal component analysis of gene expression does not reveal segregation by sex (Supplementary Fig. 1). Nevertheless, we controlled for this variable in both the network and eQTL analyses.

Since genome-wide expression studies were introduced, there has been interest in quantifying genes that are significantly differentially expressed, e.g. between failing and nonfailing states. What this linear, unitary approach fails to capture are mechanisms influencing higher order phenotypes reflected in rewiring of transcriptional partners that do not affect expression levels of specific genes. Earlier work has already led to the discovery of central genes using coexpression changes ${ }^{46,47}$. Here, we expanded this use of gene coexpression by exploiting not only gene interaction degree, but also integrated topological network differences and known pathway information. In our HF networks, we have shown how differences between these network topology properties in failing and nonfailing hearts can be used to uncover novel mechanisms and highlight new putative therapeutic targets.

\section{Methods}

Tissue collection and processing. We established a collaborative multi-institution network with a $24 / 7$ notification system and a team of travel-ready surgeons at major transplant centers to systematize the collection of cardiac tissue from failing hearts and unused heart transplant donors at operating rooms and remote locations. We put in place a series of best practices for procurement of explanted cardiac transplant tissue including harvesting explanted cardiac tissue at the time of cardiac surgery from subjects with HF undergoing transplantation and from unused donor hearts. Hearts were perfused with cold cardioplegia solution prior to cardiectomy to arrest contraction and prevent ischemic damage, and explanted cardiac tissue specimens were flash frozen in liquid nitrogen.

All samples were taken from the left-ventricular free wall at the mid ventricular level (Segments 11 or 12) on the 17 -segment model. On some of the occasions, when it was necessary to avoid infarct or peri-infarct tissue in these segments, we obtained tissues that may be closer to the base or apex or more anterior or inferior (segments 1, 4, 5, 6, 7, 10, 16). The septum was never collected. Histopathology using $\mathrm{H} \& \mathrm{E}$ and trichrome staining was used to avoid the use of donor hearts with excess fibrosis or hypertrophy. Immunostaining was not performed.

The institutional review boards at all collection sites (including Stanford, the University of Pennsylvania and the Cleveland Clinic) reviewed and approved the protocols used in this study for procurement and use of human tissue and information. All participants gave informed consent before enrollment.

Expression and genotype datasets and clinical variables. We performed RNA expression measurements and obtained genotype information in genome-wide markers for 313 patients (177 failing hearts, 136 donor, nonfailing (control) hearts) using Affymetrix expression and Affymetrix Human 6.0 respectively. Clinical variables for each individual were recorded during the course of the research and were compiled using REDCap ${ }^{48}$

\section{Data pre-processing, covariate correction, and differential expression. Several} technical and sample covariates can bias gene expression values inferred from our microarray data, such as array batch effects and individual ethnicity, gender. These covariates can greatly confound downstream analyses, resulting in false positive and negative associations and reducing the power of statistical analyses. We corrected for these biases in three ways: by normalizing to "reference" probes (control probes with a fixed fluorescence value that control for the geometry and preparation of the array), by applying batch normalization using ComBat ${ }^{49}$, and by correcting for observed covariates (gender, age, and collection site) using robust linear regression. The residuals after these corrections were then used as the gene expression values for downstream analyses. Previously to these corrections, the data was log-transformed to better resemble a normal distribution. Differential expression analysis was performed using the Significance Analysis of Microarrays $(\mathrm{SAM})^{50}$. Genes shown in Supplementary Figure 1B had the highest gene expression difference and were deemed significantly up- or downregulated with a false discovery rate of $5 \%$. QQ plots for differential expression are shown in Supplementary Fig. 4.

Coexpression networks. We ran the following methods on the covariate-corrected data described in the section above using the top $40 \%$ most variable genes $(n=$ 7960 ) and on each cohort separately, except in the case of the joint graphical LASSO (see below).

- WGCNA: We ran the WGCNA pipeline including correlation matrix, TOM transformation, and Dynamic Tree Cut module finding as prescribed ${ }^{19}$ on each network separately.

- Pearson correlation: We obtained the empirical Pearson correlation matrix and performed an absolute correlation coefficient cutoff of $a b s(R)>0.6$.

- ARACNe: We ran the method with default parameters and 500 bootstrap iterations for calculating significant gene coexpression relationships.

- Z-score: Otherwise known as the CLR method, we ran the method using the Pearson correlation matrix as input. The output is then a statistical scoring of each interaction for genes $A$ and $B$ considering all interactions of $A$ and $B$ against each other gene.

- Joint graphical LASSO (JGL): The JGL has two main parameters-a sparsity penalty to tune how many interactions are found and a group penalty that tries to match the network structure of the two cohorts. We performed a grid search on these parameters and chose the one that maximized the Akaike Information Criterion. This resulted in a very low group penalty of 0.01 and a modicum sparsity penalty of 0.1 , which was applied to a standardized matrix of expression values for both cohorts.

While in the end we used WGCNA for downstream analyses as discussed in the main text, we have made all of these networks available at https://doi.org/10.5281/ zenodo. 2600420

eQTL discovery. Prior to eQTL discovery, we used PEER to find hidden covariates that could confound signals in our data as well as filtering any genotypes with major allele frequencies less than $5 \%$. To test associations between gene expression in each cohort separately, we used QTLTools ${ }^{51}$ with an additive model accounting for gender, age, sample site, and the PEER factors as covariates. We corrected for eQTL multiple association testing using a 10,000 permutations per locus in a 2 megabase window and a false discovery rate cutoff of $5 \%$. To select the number of PEER factors, we performed the full analysis multiple times from 1 to 15 PEER factors and observed a saturation of new QTLs being discovered when using 10 factors. To intersect our variants with GTEX and the GWAS catalog, we simply matched based on rsid and position. High-resolution colocalization analysis on coronary artery disease GWAS hits was performed using the eCAVIAR pipeline (see details in Methods) ${ }^{52}$. To find independent eQTLs, we performed LD-pruning (LD, pairwise $r^{2}<0.5$ within a window of $50 \mathrm{~kb}$ ) and provide a set of pruned variants in Supplementary Data 1 and 2. QQ plots for eQTL $p$ values before and after correction for age, gender and site are provided in Supplementary Fig. 9, and were not significantly inflated by batch correction.

Cardiac GWAS colocalization analysis. We tested whether any of our eQTLs colocalized with the signals from a publicly available GWAS on coronary artery disease $^{29}$. We ran the eCAVIAR ${ }^{32}$ pipeline using the FINEMAP implementation on all loci with at least one SNP with $p<1 \mathrm{e}-5$ in the GWAS and at least one SNP with $p<1 \mathrm{e}-5$ in either condition of our eQTL study. We found evidence of colocalization at six genes: MRAS, TCF21, GPR22, LIPA, ZNF664, and EIF2B2. EIF2B2, TCF21, and ZNF664 colocalized in both failing and healthy hearts. However, LIPA colocalized only in healthy hearts, while GPR22 and MRAS colocalized only in failing hearts. These context-specific colocalizations highlight genes that may contribute to heart disease progression specifically in healthy (LIPA) or in alreadyfailing hearts (GPR22, MRAS). LIPA codes for the lysosomal enzyme lipase A. GPR22 has previously been shown to play a protective role against myocardial ${ }^{53}$. The protein MRAS is a muscle-expressed homolog of the Ras oncogene family, currently without any well-characterized mechanism in coronary artery disease.

Quantifying global and local centrality using network and community membership parameters. The local connectivity metric $(L C)$ of any gene $G$ was calculated as the difference between max-normalized weighted network degree of $G$. The global connectivity metric $(G C)$ of any gene $G$ was calculated as the number of gene sets that were significantly differentially enriched between gene rankings of failing and control networks obtained by ordering the genes by their absolute correlation coefficient to $G$.

After inferring the gene coexpression networks for both cohorts, we calculated topological properties for each gene in each network in order to get a sense of a gene's role in the networks and in the context of known pathways and gene sets. To this end, we defined a gene $g$ 's differential global connectivity $(G C)$ as the number of curated HF-relevant pathways within its neighborhood (defined by genes lying 
within a set rank of absolute edge weight to gene $g$ ) that were significantly enriched in $g$ 's neighborhood by the following procedure (see Supplementary Fig. 5):

1. For each pathway, we first ranked the neighbors of $g$ by their absolute edge weight (i.e. correlation) to $g$ in both the HF and control networks. This resulted into two ranked lists of $g$ 's neighbors: HF- and control network specific.

2. For each gene $g$, we plotted the number of network neighbors belonging to a curated list of known HF-relevant pathways from KEGG and Reactome ("HFrelevant neighbors", see Supplementary Data 9). In this analysis, the number of HF-relevant neighbors of gene $g$ in each pathway was plotted on the $y$-axis against progressively larger inclusive neighborhoods. This allowed us to create a distribution of global-HF pathway relatedness taking both known HFrelevant neighbors as well as their distance from gene $g$ into account. For example, as shown for MYH7, MYBPC3, and PPP1R3A for the KEGG HCM pathway in Fig. 3d, e, there is a steeper rise in the number of HF-relevant neighbors connected to each gene in the HF network than in the control network.

3. For each gene $g$ in each pathway, the difference between the HF network and control network curves was evaluated using the Kolmogorov-Smirnov (KS) statistic (analogous to gene set enrichment analysis ${ }^{54}$ ) with a Benjamini -Hochberg correction (false discovery rate $\mathrm{FDR}=0.01$ ).

4. A gene's GC was then defined as the number of HF-relevant pathways found to be significantly enriched in such manner.

To compare per-gene perturbations in local connectivity between HF and control networks, we defined the change in local connectivity metric $L C$ for a gene $g$ used for this purpose was calculated as follows:

$$
L C(g)=\text { deg_norm }(g \text {, HF_net }) \text { - deg_norm }(g \text {, Control_net }),
$$

where deg_norm $(g$, net) is the max-normalized weighted degree of gene $g$ in network net (sum of weights of edges that include $g$ divided by the maximum network weight across all edges).

Both $L C$ and GC are then Z-score normalized in order to call coordinator status:

- Central Coordinators have Z-score normalized $L C$ and GC greater than zero

- Local Coordinators have Z-score normalized $L C$ greater than zero but $G C$ less than zero

- Pathway Coordinators have Z-score normalized GC greater than zero but $L C$ less than zero

- Non-coordinators have neither Z-score normalized GC or $L C$ greater than zero

Isolation, culture, perturbation, and visualization of cardiac myocytes. All use of animals in this study (including the below in vivo experiments) was reviewed and approved by the Stanford University School of Medicine Institutional Animal Care and Use Committee. NRVMs were isolated from pregnant Sprague-Dawley rats (Charles River) using standard collagenase protocols as described previously and cultured in serum-free DMEM media. In order to attenuate the effects of fibroblast contamination and accentuate metabolic changes, no glucose was added to the media and a final concentration of $20 \mu \mathrm{M}$ of the fibroblast inhibitor Ara-C (Sigma-Aldrich) was incorporated. Furthermore, for robust expression measurements, at least 1 million cells were plated in a 12-well plate, corresponding to at least $70 \%$ confluency. For phenylephrine-treated cells, $50 \mu \mathrm{M}$ of phenylephrine was added $48 \mathrm{~h}$ after isolation. For the knockdown experiments, cells were transfected either with an siRNA targeted to PPP1R3A (Stealth siRNA, Invitrogen) or a scrambled siRNA using the RNAiMAX system (Invitrogen) according to the manufacturer instructions; transfections were performed $24 \mathrm{~h}$ after isolation. RNA extraction was performed using the Qiagen RNeasy kit according to the manufacturer's instructions and were DNAse-treated using the DNA-free RNA kit from Zymo research. CDNA was synthesized with the high-capacity cDNA reverse transcription kit from $\mathrm{ABI}$ and $\mathrm{qRT}$-PCR assays were performed using KAPA SYBR FAST on a ViiA 7 ABI system.

For visualization, cells were fixed directly in the culture plate wells using $4 \%$ PFA in room temperature for $10 \mathrm{~min}$, permeabilized with $0.2 \%$ Triton- $\mathrm{X}$ in room temperature for $10 \mathrm{~min}$, and blocked with DAKO protein block in room temperature for $30 \mathrm{~min}$. Afterwards, fixed cells were incubated in mouse antisarcomeric $\alpha$-actinin antibody (Sigma) in DAKO antibody buffer overnight at $4{ }^{\circ} \mathrm{C}$. Alexa secondary antibodies were added the next day after PBS washes and the cells were incubated for $1 \mathrm{~h}$ at room temperature. Cells were then washed with PBS and the nuclei were stained with DAPI (AntiFade DAPI, Invitrogen). Fluorescent cells were then imaged at $40 \times$ or $20 \times$ magnification using an Olympus BX-51 inverted fluorescent microscope. Cell area was quantified with ImageJ.

RNA sequencing and analysis pipeline. After RNA extraction, RNA integrity was checked using a 2100 BioAnalyzer (Agilent); all RNA samples had an RIN of 7.0 or higher. Samples were screened for PPP1R3A knockdown efficiency and phenylephrine treatment using qRT-PCR prior to library construction. RNAseq libraries were prepared using the TrueSeq Stranded mRNA kit (Illumina), according to the manufacturers' instruction. Libraries were barcoded, quality-checked using a 2100
BioAnalyzer and run in rapid run flow cells in a HiSeq 2500 (Illumina), producing at least 30 million paired-end reads.

Sequencing reads were aligned to the Rattus Norvegicus rn5 UCSC reference genome using the STAR aligner ${ }^{55}$. Quantification and differential expression analysis of RNAseq data was performed using the Cufflinks package ${ }^{49}$ : full transcriptome assembly was performed with Cufflinks, quantified with Cuffquant, and analyzed for differential expression using Cuffdiff. All genes deemed to be significantly up- or downregulated in the main text were called as differentially expressed by Cuffdiff.

Animals, surgery and phenotyping. Ppp1r3a $\mathrm{a}^{-/-}$mice (C57Bl6 background) were a generous gift from Anna de Paoli Roach ${ }^{41}$. Ppp $1 r 3 a^{+/+}$animals were C57Bl6 background (Jackson). All procedures involving animal use, housing, and surgeries were approved by the Stanford Institutional Animal Care and Use Committee (IACUC). Animal care and interventions were provided in accordance with the Laboratory Animal Welfare Act.

Twenty male mice (10 Ppp1r3a-/- and $10 \mathrm{Ppp} 1 \mathrm{r3} \mathrm{a}^{+/+}$) were randomized to transaortic constriction (TAC) or sham surgery (five in each group). Animals underwent $\mathrm{TAC}$ as previously described ${ }^{50}$ at 10 weeks of age. Briefly, mice were anesthetized using an isoflurane inhalation chamber, intubated and ventilated. After surgical exposure of the thoracic aorta, a 6.0 silk suture was placed between the innominate and left carotid arteries to induce a constriction of $\sim 0.4 \mathrm{~mm}$ in diameter. In sham group mice, an identical procedure was conducted, without the constriction of the aorta. One week following TAC, gradients across constriction were measured and were not different between genotypes (Ppp1r3a- ${ }^{-1-}: 32.1 \pm$ $3.9 \mathrm{mmHg}$, Ppp1r3a $a^{+/+}: 35.6 \pm 3.6 \mathrm{mmHg}, p=0.16$ ).

In vivo left-ventricular systolic function was evaluated by echocardiography in the short axis view as previously described ${ }^{50}$. Measurements occurred at 1 day prior to surgery (baseline), 7 days and 14 days after surgery and then every 14 days prior to euthanasia and tissue collection at 8 weeks after TAC.

Upon euthanasia, heart weight, body weight and tibia length were measured by standard method (Supplementary Fig. 8). Hearts were paraffin fixed, sectioned and mounted on slides. Trichrome staining as well as immunofluorescence stain for cell membrane (Rhodamine Wheat Germ Agglutinin antibody, 1:200 in PBS, Vector laboratories, Burlingame, CA) were performed for fibrosis and cell size measurements, both of which were performed using ImageJ after image capture at $20 x$.

Measurement of oxygen consumption rate. Freshly isolated NRVMs were plated in a 96-well plate at 75,000 cells/well and were maintained in kit medium with $0.5 \%$ fetal bovine syndrome. Transfection of siRNA to PPP1R3A or scrambled oligonucleotide was performed as described above 5 days after isolation. Media was changed to contain $10 \%$ fetal bovine syndrome after transfection.

Seahorse technology (XF96, Flux pack, Agilent technologies \# 10-2416100) was used to measure oxygen consumption rate (OCR) $48 \mathrm{~h}$ after transfection. Cells were either exposed to base media or media including pyruvate immediately before experiment. Basal metabolism was measured first. Maximal respiration was measured $1 \mathrm{~min}$ after delivery of $p$-triflouromethoxyphenylhydrazone (FCCP) (uncoupler of oxidative phosphorylation). After OCR measurements were complete, viable cell number was assayed using PrestoBlue Cell Viability Assay (Thermofisher \#A13261), and data were analyzed as OCR per cell.

Reporting summary. Further information on research design is available in the Nature Research Reporting Summary linked to this article.

\section{Data availability}

Data that support the findings of this study have been made available. Expression measurements for the human heart samples and clinical variables are available in GEO (accession number GSE57338). eQTLs are available at https://zenodo.org/record/ 1438557\#.W67MS5NKh24. Rat expression measurements are available via Amazon Web Services at http://s3.amazonaws.com/ashleylab-rnaseq/timecourse_analysis.tgz. All other data are contained within the article and its supplementary information or are available upon reasonable request to the corresponding author. All other source data underlying Figs. 4 and 5, and Supplementary Figs. 6 and 8 are provided in a Source Data file.

Received: 1 February 2018 Accepted: 20 May 2019

Published online: 24 June 2019

\section{References}

1. Kazi, D. S. \& Mark, D. B. The economics of heart failure. Heart Fail. Clin. 9, 93-106 (2013).

2. Eisen, H. Heart Failure: A Comprehensive Guide to Pathophysiology and Clinical Care (Springer, New York, 2017).

3. Shen, L. et al. Declining risk of sudden death in heart failure. N. Engl. J. Med. 377, 41-51 (2017) 
4. Mudd, J. O. \& Kass, D. A. Tackling heart failure in the twenty-first century. Nature 451, 919-928 (2008).

5. Chen, M. M. et al. Novel role for the potent endogenous inotrope apelin in human cardiac dysfunction. Circulation 108, 1432-1439 (2003).

6. Kittleson, M. M. et al. Gene expression analysis of ischemic and nonischemic cardiomyopathy: shared and distinct genes in the development of heart failure. Physiol. Genom. 21, 299-307 (2005).

7. Hannenhalli, S. et al. Transcriptional genomics associates FOX transcription factors with human heart failure. Circulation 114, 1269-1276 (2006).

8. van Rooij, E. et al. A signature pattern of stress-responsive microRNAs that can evoke cardiac hypertrophy and heart failure. Proc. Natl Acad. Sci. USA 103, 18255-18260 (2006)

9. Han, P. et al. A long noncoding RNA protects the heart from pathological hypertrophy. Nature 514, 102-106 (2014).

10. Haas, J. et al. Genomic structural variations lead to dysregulation of important coding and non-coding RNA species in dilated cardiomyopathy. EMBO Mol. Med. 10, 107-120 (2018)

11. Heinig, M. et al. Natural genetic variation of the cardiac transcriptome in nondiseased donors and patients with dilated cardiomyopathy. Genome Biol. 18, 170 (2017)

12. Meder, B. et al. Epigenome-wide association study identifies cardiac gene patterning and a novel cass of biomarkers for heart failure. Circulation 136, 1528-1544 (2017).

13. Margulies, K. B., Bednarik, D. P. \& Dries, D. L. Genomics, transcriptional profiling, and heart failure. J. Am. Coll. Cardiol. 53, 1752-1759 (2009).

14. The GTEx Consortium. The Genotype-Tissue Expression (GTEx) pilot analysis: multitissue gene regulation in humans. Science 348, 648-660 (2015).

15. $\mathrm{Li}, \mathrm{X}$. et al. The impact of rare variation on gene expression across tissues. Nature 550, 239-243 (2017).

16. GTEx Consortium et al. Genetic effects on gene expression across human tissues. Nature 550, 204-213 (2017).

17. Cappola, T. P. \& Margulies, K. B. Functional genomics applied to cardiovascular medicine. Circulation 124, 87-94 (2011).

18. Collado-Torres, L. et al. Reproducible RNA-seq analysis using recount2. Nat. Biotechnol. 35, 319-321 (2017).

19. Langfelder, P. \& Horvath, S. WGCNA: an R package for weighted correlation network analysis. BMC Bioinforma 9, 559 (2008).

20. Langfelder, P. \& Horvath, S. Fast R. Functions for Robust Correlations and Hierarchical Clustering. J. Stat. Softw. 46, i11 (2012).

21. Danaher, P., Wang, P. \& Witten, D. M. D. M. The joint graphical lasso for inverse covariance estimation across multiple classes. J. R. Stat. Soc. Ser. B Stat. Methodol. 76, 373-397 (2014).

22. Margolin, A. A. et al. ARACNE: an algorithm for the reconstruction of gene regulatory networks in a mammalian cellular context. BMC Bioinforma. 7 (Suppl 1), S7 (2006).

23. Zhang, B. \& Horvath, S. A general framework for weighted gene co-expression network analysis. Stat. Appl. Genet. Mol. Biol. 4, Article 17 (2005).

24. Song, L., Langfelder, P. \& Horvath, S. Comparison of co-expression measures: mutual information, correlation, and model based indices. BMC Bioinforma. 13, 328 (2012)

25. Chen, E. Y. et al. Enrichr: interactive and collaborative HTML5 gene list enrichment analysis tool. BMC Bioinforma. 14, 128 (2013).

26. Stegle, O., Parts, L., Piipari, M., Winn, J. \& Durbin, R. Using probabilistic estimation of expression residuals (PEER) to obtain increased power and interpretability of gene expression analyses. Nat. Protoc. 7, 500-507 (2012).

27. Boyle, A. P. et al. Annotation of functional variation in personal genomes using RegulomeDB. Genome Res. 22, 1790-1797 (2012).

28. Lee, J.-H. et al. Analysis of transcriptome complexity through RNA sequencing in normal and failing murine hearts. Circ. Res. 109, 1332-1341 (2011).

29. Welter, D. et al. The NHGRI GWAS Catalog, a curated resource of SNP-trait associations. Nucleic Acids Res. 42, D1001-D1006 (2014).

30. Arnold, M., Raffler, J., Pfeufer, A., Suhre, K. \& Kastenmüller, G. SNiPA: an interactive, genetic variant-centered annotation browser. Bioinformatics 31, 1334-1336 (2015).

31. Pers, T. H., Timshel, P. \& Hirschhorn, J. N. SNPsnap: a Web-based tool for identification and annotation of matched SNPs. Bioinformatics 31, 418-420 (2015).

32. Hormozdiari, F. et al. Colocalization of GWAS and eQTL Signals Detects Target Genes. Am. J. Hum. Genet. 99, 1245-1260 (2016).

33. Foster, S. R. et al. Expression, regulation and putative nutrient-sensing function of taste GPCRs in the heart. PLoS ONE 8, e64579 (2013).

34. Foster, S. R. et al. Cardiac gene expression data and in silico analysis provide novel insights into human and mouse taste receptor gene regulation. Naunyn. Schmiede. Arch. Pharmacol. 388, 1009-1027 (2015).
35. Foster, S. R. et al. Bitter taste receptor agonists elicit G-protein-dependent negative inotropy in the murine heart. FASEB J. 28, 4497-4508 (2014).

36. Alday, L. E. \& Moreyra, E. Secondary hypertrophic cardiomyopathy in infancy and childhood. Am. Heart J. 108, 996-1000 (1984).

37. Walsh, R. et al. Reassessment of Mendelian gene pathogenicity using 7,855 cardiomyopathy cases and 60,706 reference samples. Genet. Med. 19, 192-203 (2017).

38. Hubbard, M. J. \& Cohen, P. Regulation of protein phosphatase-1G from rabbit skeletal muscle. 2. Catalytic subunit translocation is a mechanism for reversible inhibition of activity toward glycogen-bound substrates. Eur. J. Biochem. 186, 711-716 (1989).

39. Newgard, C. B., Brady, M. J., O’Doherty, R. M. \& Saltiel, A. R. Organizing glucose disposal: emerging roles of the glycogen targeting subunits of protein phosphatase-1. Diabetes 49, 1967-1977 (2000)

40. Egloff, M.-P. Structural basis for the recognition of regulatory subunits by the catalytic subunit of protein phosphatase 1. EMBO J. 16, 1876-1887 (1997).

41. Suzuki, Y. et al. Insulin control of glycogen metabolism in knockout mice lacking the muscle-specific protein phosphatase PP1G/RGL. Mol. Cell. Biol. 21, 2683-2694 (2001).

42. Mori, J. et al. ANG II causes insulin resistance and induces cardiac metabolic switch and inefficiency: a critical role of PDK4. AJP: Heart Circ. Physiol. 304, H1103-H1113 (2013).

43. Huan, T. et al. A systems biology framework identifies molecular underpinnings of coronary heart disease. Arterioscler., Thromb., Vasc. Biol. 33, 1427-1434 (2013)

44. Zhao, Y. et al. Multi-omics integration reveals molecular networks and regulators of psoriasis. BMC Syst. Biol. 13, 8 (2019).

45. Savage, D. B. et al. A prevalent variant in PPP1R3A impairs glycogen synthesis and reduces muscle glycogen content in humans and mice. PLoS Med. 5, e27 (2008).

46. Dewey, F. E. et al. Gene coexpression network topology of cardiac development, hypertrophy, and failure. Circ. Cardiovasc. Genet. 4, 26-35 (2011).

47. Zhang, B., Gaiteri, C., Bodea, L. G. \& Wang, Z. Integrated systems approach identifies genetic nodes and networks in late-onset Alzheimer's disease. Cell 153, 707-720 (2013).

48. Harris, P. A. et al. Research electronic data capture (REDCap) - a metadatadriven methodology and workflow process for providing translational research informatics support. J. Biomed. Inform. 42, 377-381 (2009).

49. Trapnell, C. et al. Differential gene and transcript expression analysis of RNAseq experiments with TopHat and Cufflinks. Nat. Protoc. 7, 562-578 (2012).

50. Serpooshan, V. et al. [Pyr1]-Apelin-13 delivery via nano-liposomal encapsulation attenuates pressure overload-induced cardiac dysfunction. Biomaterials 37, 289-298 (2015).

51. Delaneau, O. et al. A complete tool set for molecular QTL discovery and analysis. Nat. Commun. 8, 15452 (2017)

52. Benner, C. et al. FINEMAP: efficient variable selection using summary data from genome-wide association studies. Bioinformatics 32, 1493-1501 (2016).

53. Adams, J. W. et al. Myocardial expression, signaling, and function of GPR22: a protective role for an orphan G protein-coupled receptor. Am. J. Physiol. Heart Circ. Physiol. 295, H509-H521 (2008).

54. Subramanian, A. et al. Gene set enrichment analysis: a knowledge-based approach for interpreting genome-wide expression profiles. Proc. Natl Acad. Sci. USA 102, 15545-15550 (2005).

55. Dobin, A. et al. STAR: ultrafast universal RNA-seq aligner. Bioinformatics 29, 15-21 (2013).

\section{Acknowledgements}

We thank members of the Ashley lab, Stanford biomedical informatics students Winn Haynes, Albee Ling, as well as other members of the NIH pre-print journal club for comments on the manuscript. The MAGNet HF study has been supported through grants for the US National Institutes of Health (HL105993, HL088577, HL098283, HL113933). This work was further supported by a predoctoral CONACyT fellowship (to P.C.), an NIH F32 postdoctoral fellowship HL134233 (to V.N.P.), an NIH T32 postdoctoral fellowship (to A.E.) and NSF Award 1564785 (to S.H.).

\section{Author contributions}

P.C., V.N.P., A.E., M.T.W., E.A.A., and T.C. designed the study. T.C., K.B.M., W.H.W.T and E.A.A. led data collection efforts with additional work from A.C.C., M.M., S.H., J.B., N.G., A.P., J.S., C.M., H.L., M.L. and S.R. P.C., D.W., M.J.G. and E.T.C. performed the computational and statistical analyses with input from F.D., S.H. and H.H. P.C, A.E, K.Z and K.S.S. performed in vitro perturbation of cardiomyocytes and time course measurements. V.N.P. performed metabolic in vitro and in vivo experiments with assistance 
from A.C.C., M.Z., C.S. and Y.H. and support from D.B. Mice were provided by A.A.D.R. P.C., V.N.P., E.T.C. and E.A.A. wrote the manuscript with input from M.T.W., S.B.M., A.J.L., T.C. and K.B.M.

\section{Additional information}

Supplementary Information accompanies this paper at https://doi.org/10.1038/s41467019-10591-5.

Competing interests: E.A.A. is founder at Personalis and DeepCell, Inc, and advisor for SequenceBio and Genome Medical. S.B.M. is on the SAB of Prime Genomics. The other authors declare no competing interests.

Reprints and permission information is available online at http://npg.nature.com/ reprintsandpermissions/

Peer review information: Nature Communications thanks Xia Yang and other anonymous reviewer(s) for their contribution to the peer review of this work.
Publisher's note: Springer Nature remains neutral with regard to jurisdictional claims in published maps and institutional affiliations.

(c) (i) Open Access This article is licensed under a Creative Commons Attribution 4.0 International License, which permits use, sharing, adaptation, distribution and reproduction in any medium or format, as long as you give appropriate credit to the original author(s) and the source, provide a link to the Creative Commons license, and indicate if changes were made. The images or other third party material in this article are included in the article's Creative Commons license, unless indicated otherwise in a credit line to the material. If material is not included in the article's Creative Commons license and your intended use is not permitted by statutory regulation or exceeds the permitted use, you will need to obtain permission directly from the copyright holder. To view a copy of this license, visit http://creativecommons.org/ licenses/by/4.0/.

(C) The Author(s) 2019 